\title{
Integrative molecular roadmap for direct conversion of fibroblasts into myocytes and myogenic progenitor cells
}

\author{
Inseon Kim ${ }^{1}$, Adhideb Ghosh ${ }^{1,3}$, Nicola Bundschuh ${ }^{1}$, Laura Hinte ${ }^{2}$, Ferdinand von Meyenn ${ }^{2}$ \& \\ Ori Bar-Nur ${ }^{*}$ \\ ${ }^{1}$ Laboratory of Regenerative and Movement Biology, Department Health Sciences and Technology, Swiss \\ Federal Institute of Technology (ETH) Zurich, Schwerzenbach, Switzerland. \\ ${ }^{2}$ Laboratory of Nutrition and Metabolic Epigenetics, Department Health Sciences and Technology, Swiss Federal \\ Institute of Technology (ETH) Zurich, Schwerzenbach, Switzerland. \\ ${ }^{3}$ Functional Genomics Center Zurich, Swiss Federal Institute of Technology (ETH) Zurich, Zurich, Switzerland. \\ *Correspondence: Ori.Bar-Nur@hest.ethz.ch
}

\section{Abstract}

Transient MyoD overexpression in concert with small molecules treatment reprograms mouse fibroblasts into induced myogenic progenitor cells (iMPCs). However, the molecular landscape and mechanisms orchestrating this cellular conversion remain unknown. Here, we undertook an integrative multi-omics approach to delineate the process of iMPC reprogramming in comparison to myogenic transdifferentiation mediated solely by MyoD. Utilizing transcriptomics, proteomics and genome-wide chromatin accessibility assays, we unravel distinct molecular trajectories which govern the two processes. Notably, iMPC reprogramming is characterized by gradual upregulation of stem and progenitor cell markers, unique signaling pathways, chromatin remodelers and cell cycle regulators which manifest via rewiring of the chromatin in core myogenic promoters. Furthermore, we determine that only iMPC reprogramming is mediated by Notch pathway activation, which is indispensable for iMPC formation and self-renewal. Collectively, this study charts divergent molecular blueprints for myogenic transdifferentiation or reprogramming and underpins the heightened capacity of iMPCs in capturing myogenesis ex vivo.

\section{Introduction:}

Skeletal muscle is a soft tissue which governs voluntary movement and accounts for $30-40 \%$ of the normal human body mass. This tissue is predominately composed of multinucleated muscle fibers that contract to generate locomotion and a variety of mononucleated resident cells that maintain tissue homeostasis ${ }^{1}$. Satellite cells are resident skeletal muscle stem cells that can regenerate muscle fibers upon injury or disease and are characterized by high expression of the transcription factor paired box protein $7(\operatorname{Pax} 7)^{2-4}$. These cells reside in a unique anatomical location between the myofiber cell membrane and basal lamina and are quiescent during homeostasis, undergoing activation to repair tissue damage following muscle insult ${ }^{2,3}$. During this repair process, activated satellite cells either divide symmetrically to increase the pool of proliferative satellite cells that eventually return to quiescence, or divide asymmetrically into myoblasts and fusion-competent myocytes that merge and repair damaged myofibers ${ }^{5}$. Satellite cell activation is a stepwise process which commences by upregulation of myogenic regulatory transcription factors including Myf5 and MyoD in myoblasts, Myogenin (Myog) and Myf6/MRF4 in myocytes and Myosin heavy chain $(\mathrm{MyHC})$ isoforms in multinucleated myofibers ${ }^{2,3}$. Following isolation from skeletal muscles and in vitro propagation, satellite cells form a population of proliferative myoblasts which upregulate MyoD and rapidly lose molecular attributes indicative of an in vivo activated satellite cell state ${ }^{6}$. This loss of satellite cell attributes following in vitro expansion renders myoblasts cumbersome for regenerative medicine purposes and highlights the necessity to seek alternative methods to culture myogenic stem and progenitor cells ${ }^{7,8}$.

Direct lineage reprogramming denotes the conversion of one cell type into another. It is typically induced by forced overexpression of cell-type-specific transcription factors or small molecule treatment ${ }^{9}$. Manipulation of cell identity via this approach was first 
demonstrated in a milestone study which determined that overexpression of the transcription factor MyoD transdifferentiates fibroblasts into skeletal muscle cells ${ }^{10}$. Since this seminal study, several works reported on transdifferentiation of somatic cells into various cell types including neurons, cardiomyocytes and hepatocytes ${ }^{11-14}$. Moreover, further works have reported on direct reprogramming of somatic cells into tissue-specific multipotent stem and progenitor cells ${ }^{15-20}$. One study recently reported on a method to directly reprogram mouse fibroblasts into "induced myogenic progenitor cells" (iMPCs) by transient MyoD overexpression in conjunction with three small molecules; the adenylate cyclase activator Forskolin (F), the TGF- $\beta$ receptor inhibitor RepSox $(R)$ and the GSK3- $\beta$ inhibitor CHIR99021 (C) (abbreviated as $F / R / C)^{20}$. Reprogramming into iMPCs is markedly different from conventional transdifferentiation into myogenic cells solely by MyoD, which typically only generates multinucleated myotubes ${ }^{20}$. In contrast, MyoD overexpression in concert with $\mathrm{F} / \mathrm{R} / \mathrm{C}$ treatment gives rise to heterogeneous and expandable myogenic cultures consisting of skeletal muscle progenitor cells that express Pax7 and Myf5, in addition to highly contractile myofiber netowork ${ }^{20}$.

The divergent lineage conversion trajectories between MyoD and MyoD+F/R/C treatment raises the question how small molecules administration endows a myogenic stem cell fate on fibroblasts in comparison to postmitotic myotubes by MyoD-mediated transdifferentiation, which has been extensively studied ${ }^{21-23}$. Furthermore, it remains unknown how molecularly akin are Pax $7^{+}$iMPCs to primary $\mathrm{Pax} 7^{+}$myoblasts. To address these questions, here we set out to delineate the molecular landscape of iMPCs utilizing integrative multi-omics approaches and further dissect the molecular trajectory guiding fibroblast conversion into iMPCs in comparison to transdifferentiation solely by MyoD. Collectively, we establish a body of knowledge in respect to the genes, proteins and pathways that govern direct lineage conversion into myogenic progenitor cells, and further establish their molecular disparity from primary myoblasts. Our results suggest that iMPCs capture a bona fide skeletal muscle differentiation program in vitro, thus establishing them as an exceptional method to culture and propagate skeletal muscle stem and progenitor cells.

\section{Results:}

\section{An inducible reprogramming system to study fibroblast conversion into myogenic cells}

We commenced our investigation by establishing an inducible cellular conversion system in fibroblasts that enables comparison of reprogramming via $M y o D+F / R / C$ to transdifferentiation solely by MyoD. To this end, we derived mouse embryonic fibroblasts (MEFs) from a transgenic mouse strain that carries a Pax7-nuclear GFP (Pax7-nGFP) reporter, which allows prospective skeletal muscle stem cell purification using fluorescenceactivated cell sorting (FACS) ${ }^{24}$. To initiate controlled MyoD overexpression in Pax7-nGFP MEFs, we engineered a novel doxycycline (dox)-inducible Tet-On lentiviral dual plasmid system harboring a MyoD coding sequence under a TRE3G promoter (tetO-MyoD/PGKPuromycin) and a Tet3G activator under the control of a constitutive EF1-a promoter (EF1artTA3/PGK-Neomycin) (Fig. 1A). Each respective plasmid also contained an antibiotic resistance gene, enabling selection of reprogrammable MEFs (Rep-MEFs) that carry both lentiviral constructs (Fig. 1A). Since MEFs might contain multiple cell types, we FACSpurified fibroblasts from MEF cultures using Thy1, a fibroblast-specific marker (Supplementary Fig. 1A) ${ }^{25}$. We then subjected Thy $1^{+}$Pax7-nGFP Rep-MEFs to either dox (MyoD) or dox+F/R/C (MyoD+F/R/C) treatment. Following 1-3 days of MyoD overexpression with or without $\mathrm{F} / \mathrm{R} / \mathrm{C}$ treatment, we documented the formation of multinucleated myotubes, however from days 6-8, Rep-MEFs treated with MyoD+F/R/C formed a highly contractile network of myofibers in conjunction with appearance of small mononucleated cells which 
proliferated robustly (Fig. 1B). To assess the expression of myogenic markers upon MyoD or MyoD+F/R/C treatment, we performed quantitative Real-Time PCR (qRT-PCR) at day 10 of reprogramming and documented robust upregulation of the myogenic stem cell markers Pax7 and Myf5 in MyoD+F/R/C treatment, whereas the differentiation marker Myog was upregulated in both conditions (Fig. 1C). Significant downregulation of the fibroblast marker Col1a1 was observed only in MyoD+F/R/C condition (Supplementary Fig. 1B).

Next, to generate stable dox-independent iMPC clones we reprogrammed Rep-MEFs using MyoD+F/R/C treatment for 10 days and then withdrew dox and expanded the cells in medium containing F/R/C for several passages, thus establishing dox-independent iMPC clones which proliferated robustly and expressed an array of myogenic progenitor and differentiation genes (Fig. 1C, D). Furthermore, these cultures contained a network of contractile myofibers as well as mononucleated Pax7-nGFP ${ }^{+}$cells, ranging between 3.622.7\%, that were also positive for the cell proliferation marker Mki67 (Fig. 1D, E, F Supplementary Fig. 1C, D). Immunostaining for MyoD and MyHC revealed the presence of $\mathrm{Pax} 7-\mathrm{nGFP}^{+} / \mathrm{MyoD}^{-} / \mathrm{MyHC}^{-}$mononucleated cells, suggesting that iMPCs contain more immature myogenic stem / progenitor cells as previously reported (Fig. 1G) ${ }^{20}$. Quantification of the cell subsets in an established iMPC clone revealed the presence of $\mathrm{Pax} 7^{+} / \mathrm{MyoD}$ (12.6 $\pm 2.5 \%), \mathrm{Pax}^{+} / \mathrm{MyoD}^{+}(4.7 \pm 1.5 \%), \mathrm{Pax}^{-} / \mathrm{MyoD}^{+}(74.9 \pm 5.3 \%)$ and $\mathrm{Pax} 7^{-} / \mathrm{MyoD}^{-}(7.9$ $\pm 7.4 \%$ ) cells, highlighting the heterogeneous cell composition of iMPCs (Fig. $1 \mathrm{H}$ ). Collectively, we successfully established a novel reprogramming system to interrogate transdifferentiation by $M y o D$ or reprogramming by MyoD+F/R/C. Furthermore, we demonstrate that subjecting fibroblasts to $M y o D+F / R / C$ treatment forms highly heterogeneous iMPC cultures consisting of mononucleated Pax7-nGFP ${ }^{+}$cells that can propagate without ectopic MyoD overexpression.

\section{Transcriptional dynamics during fibroblast conversion into skeletal muscle cells}

To gain molecular insights into the transcriptional changes which occur during iMPC formation, we opted to perform bulk RNA-Sequencing (RNA-Seq) and dissect transcriptional dynamics following MyoD or MyoD+F/R/C treatment of fibroblasts. To this end, we subjected Thy $1^{+}$Rep-MEFs to MyoD or MyoD+F/R/C treatment, followed by RNA-Seq at days 2, 4, 6, 8 , and 10 of the conversion process (Fig. 2A). As positive controls, we used iMPC clones and satellite cell-derived Pax7-nGFP primary myoblasts. Principal Component Analysis (PCA) and dendrogram clustering separated the MyoD and MyoD+F/R/C treated cells into two distinct groups, which were both transcriptionally divergent from parental MEFs (Fig. 2B, Supplementary Fig. 2A). In the PCA, a stepwise temporal trajectory was documented during MyoD+F/R/C reprogramming course, as cells gradually clustered further away from parental MEFs and became more akin to stable iMPCs and primary myoblasts (Fig. 2B). In contrast, MEFs subjected only to MyoD overexpression exhibited a haphazard temporal trajectory with no clear separation between time points (Fig. 2B). We next documented the number of differentially expressed genes (DEGs) during $M y o D$ or $M y o D+F / R / C$ conditions. This analysis revealed a prominent transcriptional wave of upregulated genes in both conditions at day 2 and 4, followed by reduced transcriptional activity during days 4-6, and an additional transcriptional wave only in MyoD+F/R/C treated MEFs at day 8 (Fig. 2C). We then investigated individual gene expression dynamics during the reprogramming course and noted upregulation of the differentiation markers Myog, Tnni1, Actn2 and Myh2 in both MyoD and MyoD+F/R/C conditions (Fig. 2D, Supplementary Fig. 2B). However, prominent expression of other key myogenic progenitor and differentiation markers including Myf5, Myf6, Six1, Six4, Myh1 and Myh4 was significantly upregulated only in MyoD+F/R/C condition (Fig. 2D, Supplementary Fig.2B). Notably, significant upregulation of immature 
myogenic stem and progenitor markers including Pax7, Dmrt2 and Sox8 was only observed in MyoD+F/R/C condition starting at day 8 of the reprogramming process (Fig. $2 \mathrm{D}$, Supplementary Fig. 2C). Additionally, pronounced downregulation of fibroblast-specific genes such as Fbln2, Col1a1 and Col5a1 was most notably observed in the presence of $\mathrm{F} / \mathrm{R} / \mathrm{C}$ treatment, whereas Thy1 expression was significantly downregulated in both conditions (Fig. 2D, Supplementary Fig. 2B, C).

We next analyzed broad transcriptional trends in the two cellular conversions via fuzzy clustering. In total, 8 gene subsets that exhibited similar expression patterns during MyoD or MyoD+F/R/C conditions were defined (Fig. 2E). The first group involved two gene sets (GS1, GS2) which were upregulated in both conditions, albeit for the MyoD condition these genes annotated with cellular processes, whereas metabolic processes were defined for the MyoD+F/R/C condition (Fig. 2E). Specifically, 266 genes in GS2 of MyoD+F/R/C treatment exhibited a gradual gene upregulation pattern over time and contained the subsets annotated with Regulation of cell cycle (Cdk2, Cdkn1c, Smc3) and Striated muscle tissue development (Pax7, Heyl, Tbx3) (Supplementary Fig. 2D, E). The second gene sets (GS3, GS4) involved downregulated genes in both conditions and in particular GS3 exhibited downregulation of the largest subsets of genes over time (Fig. 2E). Lastly, gene subsets showing transient downregulation (GS5, GS6) or upregulation (GS7, GS8) patterns were defined (Fig. 2E).

The transcriptome analysis revealed prominent gene expression differences between MyoD and MyoD+F/R/C treatment. Given this observation, we next carried out differential gene expression analysis between the two cell conversions to uncover key DEGs. The top 30 upregulated genes in $M y o D$ or MyoD+F/R/C conditions (day 10) vs. MEFs were associated with skeletal muscle differentiation markers including Trim72, Tnnt1, Tnni1, Tnnc2, Neb, Myh7, Myh8 and Actn2 (Supplementary Fig. 3A). However, the top 30 upregulated genes in MyoD+F/R/C vs. MyoD conditions at day 10 were mainly myogenic stem and progenitor cell markers including Pax7, Sox8, Msc, Lgr5, Fgfr4, Dbx1 and Heyl (Supplementary Fig. 3A). Given the elevated expression of myogenic stem cell markers in MyoD+F/R/C in comparison to MyoD at day 10 , we next performed sequential analysis of DEGs in MyoD+F/R/C vs. MyoD conditions. The most upregulated genes at days 2,4 , and 6 in $\mathrm{MyoD}+\mathrm{F} / \mathrm{R} / \mathrm{C}$ were predominantly skeletal muscle differentiation markers including Mstn, Myoz1, Myh1, Myf6 and Casq1 (Fig. 2F Supplementary Fig. 3B, 3C). The most differentially upregulated genes at a late reprogramming stage of MyoD+/F/R/C conditions (days 8 and 10) consisted of stem cell markers including Pax7, Sox8, Dbx1 and the cell cycle regulator Cdkn1c, in addition to the differentiation markers Myf6, Mstn and Myoz1 (Fig. 2F, Supplementary Fig. 3B). To further validate the expression of muscle stem cell genes during MyoD $+F / R / C$ reprogramming, we performed a meta comparison of the transcriptomes of Pax7-nGFP primary myoblasts with MEFs subjected to MyoD or MyoD+F/R/C conditions. Of note, we documented that satellite cell-associated genes are only shared between primary myoblasts and MyoD+F/R/C treated cells during days 6-10, whereas several differentiation markers were detected in all conditions for all inspected time points (Supplementary Fig. 3D). To acquire additional insights into the signaling pathways which are enriched during MyoD+F/R/C treatment, we performed pathway enrichment analysis of DEGs between MyoD+F/R/C vs. MyoD conditions using gene set enrichment analysis (GSEA) for each reprogramming time point. Pathways enriched in $M y o D+F / R / C$ condition across all time points were associated with "Cell cycle" and "Mitochondrial metabolism", whereas "Metabolism/chromatin modulation" and "Notch and Hedgehog signaling pathways" were enriched in MyoD+F/R/C condition only at days 8 and 10 (Fig. 2G, H). 
In summary, using bulk RNA-Seq we delineated transcriptional changes during cellular reprogramming manifested by $M y o D$ or $M y o D+F / R / C$ treatment and demonstrated divergent transcriptional dynamics. Solely overexpressing MyoD in fibroblasts induces fast and direct formation of multinucleated myotubes that express only a partial cohort of myogenic differentiated genes. In contrast, MyoD+F/R/C treatment entails a stepwise reprogramming process which commences with an early transcriptional wave that is characterized by upregulation of a plethora of myogenic differentiation genes prior to a second transcriptional wave that is characterized by expression of myogenic stem and progenitor cell markers. Furthermore, direct transcriptional comparison between MyoD+F/R/C and MyoD conditions at defined time points revealed canonical stem cell and differentiation markers in addition to signaling pathways that are unique to iMPC reprogramming.

\section{Proteome dynamics during direct conversion of fibroblasts into myogenic cells}

The RNA-Seq analysis revealed pronounced transcriptional differences between MEFs subjected to MyoD or MyoD+F/R/C conditions. As such, we next wished to assess whether these differences might be reflected at the protein level. To this end, we performed Liquid chromatography-mass spectrometry (LC-MS) analysis of multiple MEFs, iMPC clones as well as MEFs subjected to MyoD or $M y o D+F / R / C$ conditions at day 10 . Dendrogram clustering and PCA separated MEFs and MEFs+MyoD into one group whereas iMPCs and MyoD+F/R/C treated MEFs into another group, with further subdivision in each respective group (Fig. 3A, Supplementary Fig. 4A). To corroborate this observation further, we identified the number of differentially expressed proteins (DEPs) between the various groups. In accordance with the hierarchical clustering, the percentage of DEPs was higher for MEFs subjected to MyoD+F/R/C treatment or iMPCs than MEFs subjected to MyoD condition alone, suggesting that $M y o D+F / R / C$ manifests in more pronounced proteome changes (Fig. 3B). However, in comparison to parental MEFs we documented in all groups upregulation of several skeletal muscle associated proteins such as MYOD, DESM, DMD and TNNT1, albeit the proliferation markers PCNA and KI67 and myogenic proteins DEK and MUSK were significantly upregulated only in MyoD+F/R/C condition and stable iMPCs (Fig. 3C). We then analyzed the top 30 upregulated DEPs between each condition vs. MEFs and documented the upregulation of typical skeletal muscle proteins in all groups including MYH7, MYH8, TNNT2, ACTN3, and CASQ2 (Fig. 3D). Furthermore, a functional enrichment analysis of DEPs documented the upregulation of several skeletal muscle gene networks in all groups (Supplementary Fig. 4B). We then performed a direct comparison between MyoD+F/R/C and MyoD conditions at day 10 of reprogramming and documented 681 upregulated and 349 downregulated proteins out of 4097 total proteins (Fig. 3E). Of note, the most upregulated proteins in the $M y o D+F / R / C$ vs. MyoD conditions were associated with skeletal muscle differentiation markers and signaling proteins such as CASQ1, MYOZ1, GDF8 (Mstn), IBP2, IBP5 and cell proliferation and chromatin regulators including KI67, CDN1C, DNMT1 and BRD7 (Fig. 3F). Following MyoD+F/R/C treatment we further observed significant enrichment of cell proliferation and DNA replication proteins (MCM2, MCM3, MCM4, MCM6, PCNA, KI67), chromatin regulators (DNMT1, UHRF1, MBD3, PARP1, SETD7), signaling proteins (IGF2, TGFB2, IBP2, IBP5, CREB1) and skeletal muscleassociated proteins (PAXI, PYGM, MYH4, DEK, BCAM, CASQ1, MYOZ1) (Fig. 3G).

Next, we performed an integrative analysis between the RNA-Seq and proteome datasets. We first inspected significant DEGs and DEPs in iMPCs compared to MEFs and recorded 443 shared genes/proteins with a high correlation $(r=0.81)$ (Supplementary Fig. 4C). Notably, enriched genes/proteins in iMPCs included skeletal muscle-related markers, 
chromatin regulators and signaling pathway factors, whereas MEFs were enriched for fibroblast-specific genes and proteins (Supplementary Fig. 4C). Given this observation, a similar integrative analysis was performed for MyoD+F/R/C vs. MyoD conditions at day 10 of reprogramming and a group of 180 shared DEGs and DEPs was identified (Fig. $3 \mathrm{H})$. We noted a high correlation between the mRNA and protein levels $(r=0.78)$ and documented following $\mathrm{F} / \mathrm{R} / \mathrm{C}$ treatment a unique upregulation of skeletal muscle markers such as Dek, Igfbp2, Igfbp5, Mstn, Bcam, Myh4 and Casq1, in addition to cell proliferation and DNA replication markers such as $\mathrm{Mcm} 3, \mathrm{Mcm6}$, and Nasp (Fig. 3H). Lastly, we performed enrichment analysis for each group of upregulated and downregulated markers, thus revealing upregulation of "cell proliferation" associated networks and downregulation of "developmental processes" and "cell adhesion" associated networks following F/R/C treatment (Fig. 3I).

Collectively, the LC-MS analysis uncovered a unique expression of proteins which are significantly upregulated in iMPCs or MyoD+F/R/C condition. These proteins include muscle differentiation proteins, signaling factors, chromatin regulators and proliferation markers. The high expression of proteins such as MYOZ1, PYGM, MYH4 and DEK predominantly in the presence of $\mathrm{F} / \mathrm{R} / \mathrm{C}$ treatment suggests that iMPCs preferentially express mature muscle markers in comparison to myotubes produced via transdifferentiation by MyoD.

\section{Unique chromatin accessibility dynamics during formation of iMPCs}

Chromatin accessibility in promoter regions is well-known to be associated with increased gene expression. Given the prominent differences at the mRNA and protein levels between MyoD and MyoD+F/R/C treatment, we reasoned that divergent chromatin accessibility dynamics in promoters of key myogenic genes may explain the observed differences in the transcriptome and proteome during transdifferentiation and reprogramming. To investigate this hypothesis, we performed an Assay for Transposase-Accessible Chromatin using sequencing (ATAC-Seq) of MEFs, MEFs subjected to MyoD or MyoD+F/R/C for 2 days, and an established iMPC clone. As expected from our previous multi-omics analyses, iMPCs were characterized by a distinct chromatin accessibility profile in comparison to MEFs, and surprisingly demonstrated a high percentage of annotated peaks around promoter regions (Fig 4A, B). Given this observation, we performed differential chromatin accessibility analysis in the gene promoter regions of canonical myogenic and fibroblast genes. In comparison to MEFs, we detected a decrease of chromatin accessibility in fibroblast-specific gene promoters such as Thy1, Fbln2, Fbln5, Col1a1 and an increase in myogenic gene promoters including Myh8, DIl1, Six1 and Myod1 in all myogenic cell lines (Fig. 4C). In accordance with the transcriptome analysis, we also documented a preferential increase of chromatin accessibility in MyoD+F/R/C treated MEFs and iMPCs in multiple myogenic differentiation genes such as Myf6, Mstn and Casq1 as well as progenitor genes such as Sox8 and Fgfr4 (Fig. 4C). Notably, open chromatin configuration in the promoters of muscle stem cell markers such as Pax7, Myf5 and Notch3 was solely documented in established iMPCs (Fig. 4C).

To investigate further whether chromatin accessibility in promoter regions might be associated with differential gene expression, we performed an integrative analysis of ATACSeq and bulk RNA-Seq datasets between MyoD and MyoD+F/R/C treated MEFs at day 2, as well as established iMPCs and MEFs (Fig. 4D). For both comparisons, we noted a positive and high correlation between increased gene expression and an accessible and more "open" chromatin configuration (Fig. 4D). Of note, we detected an increase in both gene expression and chromatin accessibility in the promoter regions of myogenic genes that 
were preferentially expressed following F/R/C treatment at day 2 including Mstn, Myf6, Myh1 and Gas1 (Fig. 4D, E). Similarly, we recorded an increase of myogenic stem and differentiated cell markers unique to iMPCs in comparison to parental MEFs including Pax7, Myf5, Bcam, Heyl, Mymk, Myh1, Myh4 (Fig. 4D, E). Collectively, our results demonstrate that as early as 2 days following MyoD or MyoD+F/R/C treatment of MEFs prominent chromatin accessibility changes can be detected in myogenic promoters, however $\mathrm{F} / \mathrm{R} / \mathrm{C}$ treatment further elicits preferential open chromatin in multiple key myogenic gene promoters, which may explain their unique transcriptome and proteome profiles at this time point.

\section{Cell types and differentiation trajectories in iMPCs uncovered by single cell RNA-Seq}

The heterogeneity of iMPCs renders their entire molecular characterization challenging utilizing bulk multi-omics tools. To address this challenge and identify the underlying cell types that comprise the heterogeneous iMPC cultures, we performed single cell RNASequencing (scRNA-Seq) of an iMPC clone. Utilizing unsupervised clustering ${ }^{26}$, we identified 9 distinct cell clusters with 3 divergent cell cycle states (Fig. 5A, B). Three of these clusters $\left(1,2\right.$, and 4) represented the $\mathrm{Pax}^{+}$stem cell fraction of iMPCs, and could be further separated into cycling $(\mathrm{C} 1,16.25 \%)$ or less cycling cell populations $(\mathrm{C} 2,14.88 \%$ or $\mathrm{C} 4$, $12.58 \%$ ) (Fig. 5A-C). These clusters were also highly enriched for the myogenic stem and progenitor markers Myf5, Dek, Mest, Plagl, Dbx1, Fzd4 and Sdc4 (Fig. 5C-D, Supplementary Fig. 5A). Notably, cells in cluster 5 (C5, 10.38\%) expressed high levels of Sox8, Myod1, Myog and the canonical Notch ligand DIl1, thus representing committed progenitors, whereas late-stage differentiation markers including Myf6, Mstn, Mymk, Mymx and Myh1 were present in cluster 6, thus representing fusion-competent myocytes (C6, 7.64\%) (Fig. 5C, D, Supplementary Fig. 5A). Cells in cluster 7 (C7,6.62\%) may correspond to a more mature myofiber population given the high expression of Myh1, Tnnt3, and Tnni1 but absence of $M y o D$ or Myog (Fig. 5C, D). Surprisingly, three clusters (0, 3, and 8) highly expressed Col1a1, Thy1, Pdgfra and Pdgfrb markers which are indicative of connective tissue cells in the form of fibroblasts, mesenchymal cells or fibro-adipogenic progenitors (Fig. $5 A, C)$. Lastly, we observed that pseudo gene expression of iMPCs from scRNA-Seq strongly correlated with gene expression of iMPCs from bulk RNA-Seq, and the connective tissue-like cell populations ( $\mathrm{C} 0, \mathrm{C} 3, \mathrm{C} 8$ ) with bulk RNA-Seq of MEFs (Supplementary Fig. 5B, C). This correlation analysis collectively confirms that the cell identities revealed via scRNASeq faithfully represent the cell types we previously characterized using bulk RNA-Seq.

To investigate whether iMPCs recapitulate a myogenic differentiation program in vitro, we performed a pseudotime lineage trajectory analysis using the Monocle package ${ }^{27}$. As first step, we performed an unsupervised trajectory analysis, which revealed two major cellular progressions, both emanating from the cycling Pax $7^{+}$progenitors $(\mathrm{C} 1)$ (Fig. 5E). One branch further gave rise to several myogenic differentiated cell types $(\mathrm{C} 5, \mathrm{C} 6, \mathrm{C} 7)$. Surprisingly, another branch emanated towards a less proliferative population of $\mathrm{Pax} 7^{+}$cells and gave rise to a connective tissue-like cell type (C0). Aside from these two main trajectories, $\mathrm{C} 3$ and C8 were not associated with an active lineage progression, suggesting that these may correspond to non-reprogrammed MEFs or other mesenchymal cells (Fig. 5E).

To validate the reproducibility of the trajectory analysis, we reconstructed a cellular progression in a semi-supervised manner. Using this approach, we determined two distinct differentiation branch points (denoted as $B_{1}$ and $B_{2}$ ) and three different cell fates (denoted as $F_{1}, F_{2}$, and $F_{3}$ ) emanating from the root of trajectory $F_{0}$, which was mainly composed of $\mathrm{Pax}^{+}$progenitors (Fig. 5F, G Supplementary Fig. 5D). The $\mathrm{B}_{1}$ point represents a clear bifurcation into two distinct reprogramming routes, $F_{1}$ and $F_{3}$ (Fig. 5F). Notably, the progression from $B_{1}$ to $F_{1}$ mainly involves cells that express committed and differentiation 
myogenic genes $(C 5, C 6, C 7)$, whereas the progression from $B_{1}$ to $F_{3}$ consists of connective tissue-like cells ( $\mathrm{C} 0, \mathrm{C} 3, \mathrm{C} 8)$ (Fig. 5F, G). Lastly, we investigated the individual gene level expression in the two differentiation routes emanating from $B_{1}$. First, stem and progenitor cell markers such as Pax7 and Myf5 were highly expressed at the start of the pseudotime and were rapidly downregulated as they progressed to $F_{1}$ (Fig. $5 \mathrm{H}$, Supplementary Fig. 5E). Additionally, the committed progenitor genes Myod1 and Myog were only upregulated from $\mathrm{B}_{1}$ to $\mathrm{F}_{1}$, in conjunction with appearance of differentiation markers such as Myh1, Tnni1, Tnnt2, Tnnt3. Remarkably, DII1 and Sox 8 expressing cells were detected in the middle of the branch leading to $F_{1}$, further suggesting that this cell population represent committed myoblasts progressing to become differentiated skeletal muscle cells. In addition, the cell cycle proliferation markers Ccnb1 and Mki67 were significantly downregulated in both routes, whereas the connective tissue markers Fbln2, Thy1, Col1a1, Pdgfra and Pdgfrb were only upregulated in the path leading to $B_{2}$. Lastly, differential gene expression analysis between the two routes confirmed our observation that highly expressed genes in $\mathrm{B}_{2}$ were annotated with Extracellular matrix, whereas the genes in $\mathrm{F}_{1}$ were annotated with Synapse, Myofibril and sarcomere organization, thus reinforcing the notion that cells in $\mathrm{B}_{2}$ are skeletal muscle cells (Fig. 5I).

In summary, utilizing scRNA-Seq we identified the various cell populations that comprise the heterogeneous iMPC cultures, demonstrating the presence of cycling $P a x 7^{+}$ stem cells, Myod $1^{+} / \mathrm{Myog}^{+}$committed progenitors and $\mathrm{Myf6}^{+} / \mathrm{Myh1}^{+}$differentiated cells, in addition to connective tissue-like cells. Moreover, we reconstructed the myogenic program in an iMPC clone using a pseudotime trajectory analysis and delineated the differentiation route from activated satellite-like cells into committed progenitor cells and differentiated muscle cells. This analysis also underscored an alternative differentiation route into a connective tissue-like cell type, cautiously suggesting that iMPCs may harbor a bipotential differentiation propensity.

\section{The molecular landscape of purified Pax7-nGFP ${ }^{+}$iMPCs in comparison to myoblasts}

The scRNA-Seq analysis uncovered a unique cell population consisting of $\mathrm{Pax} 7^{+}$stem cells, however utilizing bulk RNA-Seq we could only detect a fraction of the myogenic stem cellassociated genes in this population due to the heterogeneity of iMPC cultures. Furthermore, utilizing mass spectrometry we did not detect significant levels of satellite cell and myoblastassociated proteins in bulk iMPCs. This could be due to technical limitations in utilizing LSMS to detect lowly expressed proteins in the form of stem cell-specific transcription factors due to the high expression of structural and signaling proteins emanating from the multinucleated myofibers of iMPCs.

To address this limitation and characterize the $\mathrm{Pax} 7^{+}$stem cell population in depth, we opted to FACS-purify Pax7-nGFP ${ }^{+}$cells from iMPC clones and molecularly compare them to FACS-purified Pax7-nGFP ${ }^{+}$myoblasts by bulk RNA-Seq and LC-MS (Fig. 6A). The expression levels of canonical satellite cell markers such as Pax7 and Six4 were higher in Pax7-nGFP ${ }^{+}$iMPCs and Pax7-nGFP ${ }^{+}$myoblasts, whereas the expression of skeletal muscle differentiation genes such as Myh1, Myh4, Myh8 and Casq1 was higher in bulk iMPCs, indicating that the stem cell sorting strategy from bulk iMPCs was successful (Fig. 6B). Next, to identify pathways unique to the stem cell subsets of iMPCs, we performed a pathway enrichment analysis between bulk iMPCs and Pax7-nGFP ${ }^{+}$iMPCs, thus revealing gene categories that were highly enriched in bulk iMPCs and associated with differentiated muscle cells (Fig. 6C, D). In contrast, "cell proliferation" and "metabolism/chromatin modulation" associated pathways were highly enriched in Pax7-nGFP ${ }^{+}$iMPCs in addition to the Notch and Hedgehog signaling pathways (Fig. 6C, D). 
The FACS-purification strategy further allowed us to directly compare Pax7-nGFP ${ }^{+}$ iMPCs to Pax7-nGFP ${ }^{+}$myoblasts. As expected, the two cell types were transcriptionally similar $(r=0.95)$ (Fig. 6E). However, we also documented statistically significant DEGs which included unique satellite cell markers in addition to proliferation and chromatin regulators in Pax7-nGFP ${ }^{+}$iMPCs (Fig. 6E). Further, an Over Representation Analysis (ORA) showed divergent transcriptional categories unique to Pax7-nGFP ${ }^{+}$iMPCs or myoblasts (Fig. 6F, Supplementary Fig. 6A). Strikingly, we detected high expression of activated satellite cell markers in Pax7-nGFP ${ }^{+}$iMPCs that were not detectable or lowly expressed in Pax7-nGFP ${ }^{+}$ myoblasts (Fig. 6G). These included Calcr, Musculin (Msc), Lgr5, Fos, Dmrt2, Fzd4, Gas1, Dek, Pitx3, Carm1, Sox8, Dbx1 and Plagl, many of which have been recently reported to regulate in vivo muscle regeneration and satellite cell activation ${ }^{28-36}$ (Fig. 6G). Moreover, genes associated with critical pathways for satellite cell activation and proliferation, including Notch, TGF- $\beta$, JAK-STAT and WNT were significantly upregulated in Pax7-nGFP ${ }^{+}$iMPCs (Fig. 6H). Lastly, we documented in Pax7-nGFP ${ }^{+}$iMPCs significant enrichment for chromatin remodelers, including Tet1, Tet3, Dnmt1, Dnmt3a, Dnmt3b and Uhrf1, members of the transcription factor families Myc, KLF and BEX and a plethora of cell proliferation and DNA replication markers in comparison to myoblasts (Supplementary Fig. 6B, C, D).

To examine whether the transcriptional differences could be further detected at the protein level, we opted to perform LC-MS on Pax7-nGFP ${ }^{+}$iMPCs and Pax7-nGFP $^{+}$ myoblasts. Using this approach, we detected 4444 proteins, of which 4349 were detected in both cell types (Fig. 6I). From this protein group, 508 and 310 proteins were differentially expressed in Pax7-nGFP ${ }^{+}$myoblasts and Pax7-nGFP ${ }^{+}$iMPCs, respectively (Fig. 6J). A comparison of DEPs between the two cell types documented transcription factors and chromatin regulators which were significantly more expressed in Pax7-nGFP ${ }^{+}$iMPCs in comparison to Pax7-nGFP ${ }^{+}$myoblasts including BCAM, CHD7, SIX1, DNM3A, ZEB1, FGFB2, FGFR4, KDM2A and the cell proliferation markers KI67, MCM2 and MCM3 (Fig. 6K). In summary, using a transgenic Pax7 reporter we successfully purified and characterized the transcriptome and a portion of the proteome of Pax7-nGFP ${ }^{+}$iMPCs and myoblasts. This analysis established a unique expression signature in Pax7-nGFP ${ }^{+}$iMPCs which is reminiscent of activated satellite cells and distinct from primary myoblasts.

\section{The Notch pathway is critical for iMPC formation and self-renewal}

Our transcriptional analysis thus far underpinned the involvement of the Notch pathway during iMPC formation. As such, we hypothesized that the Notch pathway is critical for iMPC formation as previously reported for proliferation of endogenous satellite cells in vivo ${ }^{37-39}$. Indeed, we detected early and rapid upregulation of Notch1, Notch3, Hey1 and Heyl only in MyoD+F/R/C during the reprogramming course (Fig. 7A). To test whether the Notch pathway is critical for iMPC formation, we treated MEFs undergoing MyoD or MyoD+F/R/C conversion with DAPT, an inhibitor of Notch target $y$-secretase ${ }^{40}$. We documented complete lack of iMPC formation and cell proliferation at day 10 of MyoD+F/R/C+DAPT treatment in comparison to MyoD+F/R/C treated cells, albeit multinucleated myotubes formed in both conditions (Fig. 7B).

To unequivocally confirm the lack of iMPC formation following DAPT treatment, we assessed the expression of Pax 7 and MyHC in MEFs subjected to MyoD+F/R/C+DAPT treatment for 10 days. Unlike MyoD+F/R/C condition, we did not detect $\mathrm{Pax} 7^{+}$cells in MyoD+F/R/C+DAPT condition, albeit $\mathrm{MyHC}^{+}$myotubes did form (Fig. 7C). In accordance with the RNA-seq data, qRT-PCR revealed that the expression of Pax7, Notch1, Hey1 and Heyl was higher in the MyoD+F/R/C than MyoD condition, however DAPT treatment abrogated the expression of these genes to levels reminiscent of MEFs or MEFs+MyoD (Fig. 7D). DAPT treatment did not alter the expression of other myogenic markers such as Myf5, 
Myod1, Mstn and Myog as well as the fibroblast-specific marker Thy1, suggesting that Notch inhibition preferentially affects the Pax $7^{+}$stem cell subset (Fig. 7D, Supplementary Fig. 7).

We next assessed the effect of Notch inhibition on the self-renewal of stable iMPC clones. To this end, we cultured iMPCs in the presence of DAPT for 5 consecutive days, observing a marked decrease in cell density and complete depletion of Pax7-nGFP ${ }^{+}$cells (Fig. 7E-G). We also confirmed the absence of $\mathrm{Pax} 7^{+}$cells via immunofluorescence and noted that $\mathrm{MyHC}^{+}$myofibers were detected in both conditions (Fig. $7 \mathrm{H}$ ). To investigate further gene regulation following DAPT treatment, we conducted qRT-PCR for myogenic and Notch-related genes in DAPT treated and non-treated iMPCs. This analysis revealed a marked reduction of Pax7 expression in concert with downregulation of Hey1, Heyl, Notch1 and Notch3 in DAPT treated iMPCs, whereas Myod1, Myog, and Mstn were expressed in similar fashion (Fig. 7I).

Next, we analyzed the expression level of Notch pathway-associated genes in the various cell subsets comprising a stable iMPC clone utilizing the scRNA-Seq dataset (Fig 5). In accordance with the bulk RNA-Seq analysis of FACS-purified Pax7-nGFP ${ }^{+}$iMPCs, we documented Pax $7^{+}$cells which highly expressed the Notch receptors Notch3 and Notch1, in addition to their canonical downstream targets Heyl and Hey1 (Fig. 7J). Strikingly, the cell subset of Sox $8^{+} / \mathrm{MyoD}^{+}$progenitor cells and $\mathrm{MyHC}^{+} / \mathrm{Myog}^{+}$differentiated cells expressed the canonical Notch ligands DII1, Jag2 and DIk1 as well as the Notch inhibitors Cdkn1c and Hes6 (Fig. 7J). This observation suggests a crosstalk via the Notch pathway between the stem cells and progenitor / differentiated cell subsets in iMPCs.

Collectively, we surmise that activation of the Notch pathway is a unique feature of $\mathrm{Pax}^{+}$cell formation during MyoD+F/R/C reprogramming. DAPT-mediated Notch pathway inhibition during MyoD+F/R/C reprogramming and in iMPC clones precludes the formation of $\mathrm{Pax}^{+}$cells and derails their self-renewal. However, myocytes and myotubes can still form following DAPT treatment, suggesting that Notch inhibition specifically prevents the formation of myogenic stem cell during iMPC reprogramming, and their maintenance in established iMPC clones. Lastly, stable iMPCs contain both $\mathrm{Pax} 7^{+}$stem cells that express canonical Notch markers in addition to their differentiated progeny which express Notchassociated ligands and inhibitors, thus recapitulating in vitro their expression during in vivo muscle regeneration.

\section{Discussion:}

Direct lineage reprogramming of somatic cells into multipotent stem or progenitor cells affords an attractive approach to generate desired cell types for basic research or therapeutic applications. This approach entails several advantages in comparison to canonical transdifferentiation, which typically involves direct conversion of one differentiated cell type into another. Namely, directly reprogrammed progenitors may exhibit self-renewal and multipotency, rendering them more attractive for cell-based therapies. However, most studies to date have reported on protocols to directly transdifferentiate cells, and only a handful of studies documented direct conversion of somatic cells into multipotent progenitors $^{9}$. Furthermore, numerous studies have characterized the molecular transitions and mechanisms governing transdifferentiation, yet very few interrogated how the molecular landscape metamorphoses during direct conversion into multipotent progenitor cells ${ }^{9}$.

In this study we set out to address this objective using the skeletal muscle lineage as a model system. To this end, we dissected the molecular changes that accompany fibroblast conversion into myogenic stem and progenitor cells by way of sustained MyoD overexpression in concert with administration of the three small molecules Forskolin, RepSox and CHIR99210 (F/R/C) ${ }^{20}$. Utilizing multi-omics approaches, we contrasted this lineage conversion to that of canonical MyoD-mediated transdifferentiation and delineated 
an array of genes, proteins and signaling pathways that are unique to each cell fate conversion. We demonstrated that reprogramming to iMPCs occurs via a gradual, stepwise reprogramming process, whereas transdifferentiation into myotubes is typically fast and direct. Additionally, we report that the two cell conversions share phenotypical characteristics, including rapid upregulation of several skeletal muscle differentiation genes, albeit are also distinct, as only $\mathrm{F} / \mathrm{R} / \mathrm{C}$ administration manifests a robust upregulation of satellite cell genes and key skeletal muscle differentiation markers via rewiring of the chromatin in promoters of key myogenic genes. We then further compared the transcriptomes and proteomes of FACS-purified Pax7-nGFP ${ }^{+}$iMPCs to that of Pax7-nGFP ${ }^{+}$primary myoblasts and documented their congruent and divergent molecular traits. Lastly, we identified Notch as a molecular pathway that is absolutely essential for the formation of iMPCs in addition to governing their self-renewal.

Several observations emanate from this study. One notable finding pertains to the molecular comparison of Pax7-nGFP ${ }^{+}$iMPCs to that of Pax7-nGFP ${ }^{+}$myoblasts. These two cell types share molecular attributes at the mRNA and protein levels, however Pax7-nGFP ${ }^{+}$ iMPCs also uniquely expressed a cohort of genes which are indicative of satellite cell activation and proliferation in vivo, in addition to increased expression of cell cycle regulators and unique signaling pathways. We postulate several reasons which may account for these disparities. First, the Pax7-nGFP ${ }^{+}$iMPCs are cultivated in the vicinity of neighboring committed progenitors, connective tissue-like cells and a highly contractile myofiber network, in contrast to primary myoblasts which are typically cultured as dispersed mononucleated cells that are passaged before fusion into myotubes. As such, the heterogeneity of iMPCs may recapitulate the microenvironment activated satellite cells encounter during skeletal muscle regeneration in vivo. Moreover, recent works have reported a crosstalk between resident muscle cells, myofibers and satellite cells during homeostasis and regeneration ${ }^{41-44}$. Similarly, signaling molecules secreted from the multinucleated myofibers of iMPCs may affect the gene expression of Pax7-nGFP ${ }^{+}$iMPCs, potentially rendering them more akin to activated satellite cells. An additional explanation for the discrepancies between iMPCs and myoblasts involves the different culture conditions used to cultivate these cells. Whereas primary myoblasts were cultured on matrigel coated plates and in medium containing bFGF and high serum ${ }^{45}$, the iMPCs were cultured directly on plastic dishes using cell medium containing Serum Replacement, Serum, bFGF and most notably F/R/C. These cell media supplements may affect molecular attributes and elicit repression or activation of multiple myogenic genes and signaling pathways. In accordance with this hypothesis, exposure of dissociated skeletal muscle tissue fragments to iMPC media containing F/R/C treatment facilitated the formation of heterogeneous cultures consisting of myogenic progenitor cells and a contractile myofiber network that resemble fibroblast-derived iMPCs ${ }^{20}$.

Direct reprogramming using $M y o D+F / R / C$ triggers extensive molecular transformations. One notable pathway that was uniquely upregulated following $\mathrm{F} / \mathrm{R} / \mathrm{C}$ treatment is Notch. This pathway has been extensively studied in the context of satellite cell quiescence and activation, and was shown to be critical for their self-renewal ${ }^{32,46-48}$. In this study, we demonstrate that this pathway is redundant for myotube formation, however, is critical for the generation of Pax $7^{+}$iMPCs and their maintenance. Moreover, we documented a high expression of Notch pathway-associated genes such as Notch3, Heyl and Hey1 in the $\mathrm{Pax} 7^{+}$subset of iMPCs, whereas Notch ligands such as DII1, Jag2 and DIk1 were expressed in downstream differentiated myoblasts and myocytes. This observation is reminiscent of the Notch receptor / ligand interaction in vivo, suggesting a potential role for the differentiated cell subset of iMPCs in triggering elevated Notch levels in the Pax $7^{+}$cell subset. It will be of further interest to compare the levels of the Notch intra-cellular domain (NICD) in iMPCs in addition to culturing them on Notch ligands to assess whether such treatment may elicit a higher expression of the Notch pathway genes and increase the self-renewal of $\mathrm{Pax} 7^{+}$cells 
as previously shown for satellite cells ${ }^{49,50}$. How each small molecule exerts its effect during reprogramming or supports the proliferation of established iMPC clones is yet to be fully explored. Previous works have established the effect of Forskolin on enhancing myoblast proliferation and engraftment capacities in mice, and TGF- $\beta$ inhibition was recently shown to promote myoblast fusion ${ }^{51-53}$. When administered together, these molecules have also been reported to enhance satellite cell quiescence in a $3 \mathrm{D}$ skeletal muscle tissue bioconstruct ${ }^{54}$. It is of interest to further explore their individual effects during reprogramming and whether $\mathrm{F} / \mathrm{R} / \mathrm{C}$ administration may augment the propensity of satellite cells to repair skeletal muscles in vivo.

To date, the conversion of somatic cells into iMPCs has only been reported from WT fibroblasts. As iMPCs recapitulate a unique myogenic differentiation program in vitro, it will be of interest to attempt their establishment from fibroblasts of murine models for muscular dystrophies. Namely, generation of iMPCs from a mouse model for Duchenne muscular dystrophy can provide a population of proliferative progenitors and contractile myofibers that lack dystrophin expression, affording a novel mean to model disease pathology in vitro, a platform for drug screens or a cell source for autologous cell therapy following genetic correction. Finally, several recent studies reported on direct conversion of mouse somatic cells into iMPCs using a variety of transcription factors and small molecules ${ }^{18-20}$. Reports on the conversion of human fibroblasts directly into myogenic stem and progenitor cells with satellite cell attributes and robust engraftment capacities are still lacking, and further work is highly warranted to establish such cell lines. We envision that with success, human iMPCs could serve as a complimentary toolbox in the skeletal muscle field for basic research, disease modeling and as a potential source for cell-based therapies.

\title{
Acknowledgments:
}

We wish to thank all members of the Regenerative and Movement Biology laboratory for their constructive comments and feedback. We are grateful to Dr. Shahragim Tajbakhsh for providing the Pax7-nGFP mouse strain and Dr. Katrien De Bock for fruitful discussions. We acknowledge the use of the Functional Genomics Center Zurich (FGCZ) facility and infrastructure and are grateful to Lennart Opitz and Laura Kunz for their assistance with data analysis of RNA sequencing and LC-MS.

\section{Funding}

Eccellenza Grant of the Swiss National Science Foundation PCEGP3_187009 (OBN)

The Good Food Institute Foundation of the Swiss National Science Foundation 1-007082 (OBN)

The Novartis Foundation for Medical-Biological Research of the Swiss National Science Foundation 1-006449 (OBN)

The Helmut Horten Foundation of the Swiss National Science Foundation 1-007211 (OBN) The National Centre of Competence in Research (NCCR) Robotics of the Swiss National Science Foundation 5-29626 (OBN)

The European Research Council (ERC) under the European Union's Horizon 2020 research and innovation program 803491 (FVM)

\author{
Author contributions \\ Experiment design: IK, AG, OBN \\ Conceptualization: IK, OBN \\ Investigation: IK, AG \\ Methodology: IK, NB, LH \\ Supervision: OBN, FVM \\ Writing - original draft: OBN, IK \\ Writing - review \& editing: IK, AG, NB, LH, FVM, OBN
}




\section{Competing interests}

The authors declare that they have no competing interests.

\section{Data and materials availability}

Plasmids generated in this study can be obtained from the Lead Contact or VectorBuilder (ID VB170530-1031pbc, ID VB181022-1110vfj). The bulk RNA-Seq and scRNA-Seq datasets generated in this study are available in Gene Expression Omnibus (GEO) repository (GSE169053 and GSE169054, respectively).

\section{Methods:}

\section{Animals}

Mice carrying a Pax7-nGFP reporter (Tg:Pax7-nGFP/C57BL6;DBA2) were previously generated $^{55}$. All mice used in this study were housed with 3-4 littermates and maintained under specific-pathogen-free (SPF)-like conditions. Mice were fed standard food and water and handled in accordance with the Swiss Federal Law on Animal Protection. All animal procedures were approved by the Zürich Cantonal Animal Welfare Committee (license ZH108/18).

\section{Plasmid construction}

The plasmids used in this study were generated by VectorBuilder. LV-EF1a-rtTA3/PGKNeomycin and LV-tetO-MyoD/PGK-Puromycin denote the respective plasmids: $p L V$ [Exp]Neo-EF1A>Tet3G (VectorBuilder, VB170530-1031pbc) and pLV[Tet]-PuroTRE3G>mMyod1[NM_010866. 2] (VectorBuilder, VB181022-1110vfj).

\section{Virus production and storage}

About $60-70 \%$ confluent HEK-293T cells were prepared in a $15 \mathrm{~cm}$ culture plate. For plasmid transfection, $\Delta 8.9(16.5 \mu \mathrm{g})$, VSV-G envelope $(11 \mu \mathrm{g})$ and $22 \mu \mathrm{g}$ of the target plasmid (either pLV[Exp]-Neo-EF1A>Tet3G or pLV[Tet]-Puro-TRE3G>mMyod1) were mixed in $150 \mathrm{mM}$ $\mathrm{NaCl}$ at a final volume of $1 \mathrm{~mL}$, followed by 10 mins incubation with $1 \mathrm{~mL}$ of $2 \mathrm{mg} / \mathrm{mL}$ Polyethylenimine (PEI) (Polysciences, POL23966-1). As next step, HEK-293T cells were incubated with a DNA/PEI mixture solution. After 1 day, the DNA/PEI mixture was replaced with fresh medium. At days 2 and 3 , medium containing virus was collected and filtered through a $0.45 \mu \mathrm{m}$ syringe filter (Corning, 431220). For virus precipitation, filtered medium was incubated with 5X PEG-it solution (System Biosciences, LV825A-1) overnight and centrifugated at $1500 \mathrm{xg}$ for 30 mins at $4^{\circ} \mathrm{C}$. The virus pellet was then re-suspended in PBS (Thermo Fisher Scientific, 10010015) containing $25 \mathrm{mM}$ HEPES buffer (Thermo Fisher Scientific, 15630056 ) in $1 / 10$ to $1 / 100$ of original volume and stored at $-80^{\circ} \mathrm{C}$.

\section{Generation of Thy $1^{+}$reprogrammable MEFs (Rep-MEFs)}

Mouse embryonic fibroblasts (MEFs) were isolated from Pax7-nGFP mice and cultured in 'MEF medium' containing high glucose DMEM (Thermo Fisher Scientific, 41966029) supplemented with 10\% FBS (Thermo Fisher Scientific, 10270106), 1\% GlutaMAX (Thermo Fisher Scientific, 35050061), 1\% non-essential amino acids (Thermo Fisher Scientific, 11140050), 1\% Pen-Strep (Thermo Fisher Scientific, 15140122) and $0.1 \% 2$ Mercaptoethanol (Thermo Fisher Scientific, 21985023). To generate dox-inducible reprogrammable MEFs (Rep-MEFs), cells were passaged in a 6 well plates and once confluent transduced with LV-EF1a-rtTA3/PGK-Neomycin plus LV-tetO-MyoD/PGKPuromycin at $1: 1$ ratio and supplemented with $6 \mu \mathrm{g} / \mathrm{mL}$ of polybrene (Sigma-Aldrich, TR- 
1003-G). Transduced MEFs were expanded and selected by sequential antibiotics treatment with MEF medium containing either $1 \mathrm{mg} / \mathrm{mL}$ of G-418 solution (Sigma-Aldrich, 4727878001) or $1 \mu \mathrm{g} / \mathrm{mL}$ of Puromycin (Thermo Fisher Scientific, A1113803) for a total of 4 days. To establish a homogenous population of fibroblasts from Rep-MEF cultures, we FACS-purified these cultures with an antibody recognizing the fibroblast-specific surface marker CD90.2 (Thy 1.2) (Thermo Fisher Scientific, 48-0902-80). Cells were FACS-purified or analyzed using an SH800S FACS-Sorter (Sony Biotechnologies).

\section{Reprogramming of Thy $1^{+}$Rep-MEFs}

Approximately $2.5 \times 10^{5}-3.0 \times 10^{5}$ Thy $1^{+}$MEFs were seeded onto 6 well plates and treated by means of the following conditions 1 day post seeding. For MyoD condition, Thy $1^{+}$Rep-MEFs were cultured with $2 \mu \mathrm{g} / \mathrm{mL}$ of doxycycline (dox) (Sigma-Aldrich, D9891) in 'iMPC medium' containing KnockOut DMEM (Thermo Fisher Scientific, 10829018) supplemented with 10\% KnockOut Serum Replacement (Thermo Fisher Scientific, 10828028), 10\% FBS, 1\% GlutaMAX, 1\% non-essential amino acids, 1\% Pen-Strep, 0.1\% 2-Mercaptoethanol and 10 $\mathrm{ng} / \mathrm{mL}$ basic FGF (R\&D Systems, 233-FB). For MyoD+F/R/C condition, Thy ${ }^{+}{ }^{+}$Rep-MEFs were cultured in 'iMPC medium' containing $2 \mu \mathrm{g} / \mathrm{mL}$ of dox and administrated three small molecules: $5 \mu \mathrm{M}$ of Forskolin (F) (R\&D Systems, 1099/50), $5 \mu \mathrm{M}$ of RepSox (R) (R\&D Systems, 3742/50) and $3 \mu \mathrm{M}$ of CHIR99021 (C) (R\&D Systems, 4423/50).

\section{Establishment and maintenance of stable iMPC clones}

To establish iMPC clones, Thy $1^{+}$Rep-MEFs were cultured in MyoD+F/R/C condition for 10 days, followed by another 2-3 days of culture in iMPC medium that contains only Forskolin, RepSox and CHIR99210 (without dox). For maintenance of stable clones, P0 iMPC clones were trypsinized and further expanded in iMPC medium containing the three small molecules.

\section{Satellite cell isolation}

Whole body skeletal muscles were harvested from Pax7-nGFP mice and minced thoroughly with surgical scissors. PBS was added to minced muscles, followed by centrifugation at 350 $\mathrm{xg}$ for 2 mins. Cell pellets were incubated with $0.2 \%$ Collagenase type 2 (Thermo Fisher Scientific, 17101015) in high glucose DMEM for 90 mins in a $37^{\circ} \mathrm{C}$ shaking water bath. After collagenase digestion, cell pellets were washed once with "wash buffer" consisting of Ham's F-10 Nutrient mix (Thermo Fisher Scientific, 22390025) supplemented with 10\% Horse serum (Thermo Fisher Scientific, 16050122). This was followed by Dispase digestion with F10 containing $0.4 \%$ Dispase II (Thermo Fisher Scientific, 17105041) and 0.2\% Collagenase type 2 for 30 mins in a $37^{\circ} \mathrm{C}$ shaking water bath. After 30 mins incubation, remaining cells were filtered and washed several times. The final cell pellet was re-suspended in PBS containing $2 \%$ FBS and satellite cells carrying Pax7-nGFP reporter were purified using an SH800S FACS-Sorter (Sony Biotechnologies).

\section{Myoblast culture}

Myoblasts were seeded onto Matrigel-coated plates and cultured in 'Myoblast medium'. To prepare Matrigel-coated plates, 4\% of Matrigel (Corning, 356237) diluted in low glucose DMEM (Thermo Fisher Scientific, 31885023) was applied to the plate which was placed on ice. After 7 mins of incubation on ice, Matrigel was removed from the plate which was further incubated for $1 \mathrm{hr}$ at $37^{\circ} \mathrm{C}$. For 'Myoblast medium', high glucose DMEM and F-10 were mixed at a similar ratio and supplemented with $20 \%$ FBS, $10 \%$ horse serum, $1 \%$ Pen-Strep 
and $10 \mathrm{ng} / \mathrm{ml}$ basic FGF as previously described ${ }^{56}$. Only P2-P4 myoblasts were used for the reported analyses.

\section{Quantitative Real time-PCR (qRT-PCR)}

Total RNA was extracted using RNeasy mini kit (Qiagen, 74104). The RNA was subjected to DNase treatment (Qiagen, 79254) and its concentration was measured with a Tecan plate reader. cDNAs were synthesized using a High-Capacity cDNA Reverse Transcription Kit (Thermo Fisher Scientific, 4368814) according to the manufacturer's protocol. qRT-PCR was performed in a $10 \mu \mathrm{L}$ reaction containing $10 \mathrm{ng}$ of cDNA, $5 \mu \mathrm{L}$ of Applied Biosystems PowerUp SYBR Green Master Mix (Thermo Fisher Scientific, A25741) and 0.4 $\mu \mathrm{L}$ of each $10 \mu \mathrm{M}$ forward and reverse primer for the target genes. Pgk was used as a house-keeping gene. The sequence of the primers for each target gene is described in Table S1.

Table S1. Sequences of the primers used for qRT-PCR

\begin{tabular}{|c|c|c|}
\hline Gene & Direction & Sequence $5^{\prime}$-> 3' \\
\hline \multirow{2}{*}{ Pax7 } & Forward & GACGACGAGGAAGGAGACAA \\
\hline & Reverse & ACATCTGAGCCCTCATCCAG \\
\hline \multirow{2}{*}{ Myf5 } & Forward & AAGGCTCCTGTATCCССTCAC \\
\hline & Reverse & TGACCTTCTTCAGGCGTCTAC \\
\hline \multirow{2}{*}{ Myod1 } & Forward & CCACTCCGGGACATAGACTTG \\
\hline & Reverse & AAAAGCGCAGGTCTGGTGAG \\
\hline \multirow{2}{*}{ Myog } & Forward & GAGACATCCCCCTATTTCTACCA \\
\hline & Reverse & GCTCAGTCCGCTCATAGCC \\
\hline \multirow{2}{*}{ Myf6 } & Forward & AGATCGTCGGAAAGCAGC \\
\hline & Reverse & CCTGGAATGATCCGAAACAC \\
\hline \multirow{2}{*}{$\mathrm{MyHC}$} & Forward & GCGAATCGAGGCTCAGAACAA \\
\hline & Reverse & GTAGTTCCGCCTTCGGTCTTG \\
\hline \multirow{2}{*}{ Pgk } & Forward & ATGTCGCTTTCCAACAAGCTG \\
\hline & Reverse & GCTCCATTGTCCAAGCAGAAT \\
\hline \multirow{2}{*}{ Thy 1} & Forward & ССТ TAC CCT AGC CAA CTT CAC \\
\hline & Reverse & AGG ATG TGT TCT GAA CCA GC \\
\hline \multirow{2}{*}{ Col1a1 } & Forward & GCA ACA GTC GCT TCA CCT AC \\
\hline & Reverse & GTG GGA GGG AAC CAG ATT G \\
\hline \multirow{2}{*}{ Notch1 } & Forward & CAGGAAAGAGGGCATCAG \\
\hline & Reverse & AGCGTTAGGCAGAGCAAG \\
\hline \multirow{2}{*}{ Notch3 } & Forward & GTCCAGAGGCCAAGAGACTG \\
\hline & Reverse & CAGAAGGAGGCCAGCATAAG \\
\hline \multirow{2}{*}{ Hey1 } & Forward & TGAATCCAGATGACCAGCTACTGT \\
\hline & Reverse & TACTTTCAGACTCCGATCGCTTAC \\
\hline \multirow{2}{*}{ HeyL } & Forward & CAGATGCAAGCCCGGAAGAA \\
\hline & Reverse & ACCAGAGGCATGGAGCATCT \\
\hline
\end{tabular}




\begin{tabular}{l|l|l|}
\multirow{3}{*}{ Mstn } & Forward & CAGGAGAAGATGGGCTGAATC \\
\cline { 2 - 3 } & Reverse & GGAGTGCTCATCGCAGTCAA \\
\hline
\end{tabular}

\section{Immunofluorescence (IF) staining and imaging}

Cells were fixed by $4 \%$ paraformaldehyde (PFA) (Alfa Aesar, 43368) for 10 mins and incubated in blocking PBS solution containing 2\% BSA (AppliChem, 9048-46-8) and 0.2\% Triton $^{\mathrm{TM}}$ X-100 (Sigma-Aldrich, 9002-93-1) for 30 mins to permeabilize cell membrane and block unspecific antigen binding. As next step, the cells were incubated with primary antibodies in blocking solution for $2 \mathrm{hrs}$, followed by 30 mins incubation with secondary antibodies and DAPI for nuclei staining (1:1000, Thermo Fisher Scientific, 62248). Stained cells were imaged using a Nikon microscope (ECLIPSE Ti2). The following primary antibodies have been used in this study: Anti-Human/Mouse/Rat/Chicken Pax7 $(5 \mu \mathrm{g} / \mathrm{ml}$, R\&D Systems, MAB1675), Anti-Human/Mouse Myod1 (5.8A) (1:100, Thermo Fisher Scientific, MA512902), Anti-Mouse Myosin Heavy Chain (1:1000, R\&D Systems, MAB4470) and Anti-Ki-67 (SP6) (1:250, Thermo Fisher Scientific, MA514520). The following secondary antibodies were used in this study at 1:500 dilution: Anti-Mouse lgG1 (Goat, Alexa Fluor 647) (Thermo Fisher Scientific, A21240), Anti-Mouse IgG2B (Goat, Alexa Fluor 546) (Thermo Fisher Scientific, A21240) and Anti-Rabbit IgG (H+L) (Donkey, Alexa Fluor 546) (Thermo Fisher Scientific, A10040).

\section{Immunofluorescence image quantification}

The quantification of immunofluorescent images was performed by counting positive and negative cells. The percentage of the positive cells for each protein was calculated by normalizing the number of positive cells by that of DAPI positive cells.

\section{Inhibition of Notch via DAPT treatment during reprogramming}

For assessment of DAPT treatment during MEF reprogramming, $2.5 \times 10^{5}-3.0 \times 10^{5}$ of RepMEFs were seeded on 6 well plates and exposed to MyoD or MyoD+F/R/C conditions. For the MyoD+F/R/C+DAPT condition, DAPT (Sigma-Aldrich, D5942) was additionally added to the MyoD+F/R/C condition at a final concentration of $15 \mu \mathrm{M}$. All analyses associated with MEF reprogramming in the presence of DAPT were performed at day 10 of treatment. For iMPC cultures, identical number of iMPCs were seeded onto 24 wells plate and cultured in 'iMPC medium' either without DAPT (-DAPT) or with DAPT (+DAPT) at the same concentration and conducted 5 days after DAPT treatment began.

\section{Bulk RNA-Sequencing (RNA-Seq)}

Total RNA was extracted using RNeasy mini kit according to the manufacturer's protocol. The RNA quality was determined by measuring the $28 \mathrm{~s} / 18 \mathrm{~s}$ ratio with a Fragment Analyzer (Agilent, Santa Clara, California, USA). Samples with a $28 \mathrm{~S} / 18 \mathrm{~S}$ ratio over 1.9 were used for library preparation. Libraries were constructed according to the TruSeq Stranded mRNA protocol (Illumina, Inc., California, USA). Briefly, 100-1000 ng of total RNA was poly-A enriched and reverse-transcribed into double-stranded cDNA. Then, the cDNA was fragmented, end-repaired and adenylated before ligation of TruSeq adapters containing unique dual indices (UDI) for multiplexing. Fragments containing TruSeq adapters on both ends were selectively enriched with PCR. The quality and quantity of the enriched libraries were validated using a Fragment Analyzer, which resulted in an average fragment size of approximately $360 \mathrm{bp}$. The libraries were normalized to $10 \mathrm{nM}$ in Tris-Cl buffer $(10 \mathrm{mM}$, pH8.5) with $0.1 \%$ Tween 20 and sequenced with $100 \mathrm{bp}$ single end reads on Novaseq 
6000 (Illumina, Inc., California, USA) according to standard protocol. Around 20 million reads were obtained for each sample.

\section{Bulk RNA-Seq data analysis}

Raw sequencing reads were pre-processed using the SUSHI framework which was developed in the Functional Genomics Center Zurich $^{57}$ The reads were trimmed (adapter sequences, low quality end and low-quality reads with phred score < 20) first using Trimmomatic $v 0.36^{58}$. Pseudoalignment of the trimmed reads was performed against the reference mouse genome assembly GRCm38.p6 and gene expression level (GENCODE release 23) was quantified using Kallisto v0.44 ${ }^{59}$. Differential gene expression analysis was conducted between different time points as well as different conditions based on negative binomial distribution using the $R$ package edgeR v3.2860. Genes showing variable expression with adjusted (Benjamini-Hochberg method) $p$-value $<0.05$ and two-fold change were considered to be differentially expressed. Gene expression time series data were subjected to soft clustering ${ }^{61}$ using the $\mathrm{R}$ package Mfuzz $\mathrm{v} 2.50^{62}$ to reveal the underlying hidden expression patterns in MyoD and MyoD+F/R/C conditions. For each fuzzy cluster, genes were annotated by gene ontology (GO) terms using the STRING v11 ${ }^{63}$ database.

\section{Protein extraction and digestion}

Cultured cells were collected using a cell scraper, snap-frozen in dry ice for 30 mins and stored at $-80^{\circ} \mathrm{C}$. For protein extraction and digestion, cell pellets were lysed by using a commercial iST Kit (PreOmics, Germany) according to the updated version of the manufacturer's protocol. Briefly, $100-200 \mu \mathrm{g}$ of the cell pellets were solubilized in 'Lyse' buffer, boiled at $95^{\circ} \mathrm{C}$ for 10 mins and processed with High Intensity Focused Ultrasound (HIFU) for 30 s under the ultrasonic amplitude to $85 \%$. Then cell lysates were transferred to the cartridge and digested by adding $50 \mu \mathrm{L}$ of the 'Digest' solution. After an hour of incubation at $37^{\circ} \mathrm{C}, 100 \mu \mathrm{L}$ of 'Stop' solution was added to halt protein digestion. The cartridge was centrifuge at $3800 \mathrm{xg}$ and the through-put was discarded. The peptides remaining in the iST-filter of the cartridge were washed, eluted, dried and re-solubilized in 20 $\mu \mathrm{L}$ of 'LC-Load' buffer for MS-Analysis.

\section{Liquid chromatography-mass spectrometry (LC-MS) analysis}

Mass spectrometry analysis was performed on an Orbitrap Fusion Lumos (Thermo Fisher Scientific) equipped with a Digital PicoView source (New Objective) and coupled to a MClass UPLC (Waters). Channel A was filled with $0.1 \%$ formic acid in water while channel B filled with $0.1 \%$ formic acid and $99.9 \%$ acetonitrile. For each sample, $1-2 \mu \mathrm{L}$ of peptides were loaded on a commercial MZ Symmetry C18 Trap Column (100A, $5 \mu \mathrm{m}, 180 \mu \mathrm{m} \times 20 \mathrm{~mm}$, Waters) followed by nanoEase MZ C18 HSS T3 Column (100A, $1.8 \mu \mathrm{m}, 75 \mu \mathrm{m} \times 250 \mathrm{~mm}$, Waters). After 3 mins of initial hold at $5 \%$ B, a gradient from 5 to $22 \%$ B in 83 mins and 22 to $32 \%$ B in additional 10 mins was applied. The column was cleaned after the run by increasing to $95 \%$ B and holding $95 \%$ B for 10 mins prior to re-establishing loading condition. Samples were acquired in a randomized order. The mass spectrometer was operated in data-dependent mode (DDA) acquiring a full-scan MS spectrum $(300-1,500 \mathrm{~m} / \mathrm{z})$ at a resolution of 120,000 at $200 \mathrm{~m} / \mathrm{z}$ after accumulation to a target value of 500,000 . Datadependent MS/MS were recorded in the linear ion trap using quadrupole isolation with a window of $0.8 \mathrm{Da}$ and HCD fragmentation with $35 \%$ fragmentation energy. The ion trap was operated in rapid scan mode with a target value of 10,000 and a maximum injection time of 50 ms. Only precursors with intensity above 5,000 were selected for MS/MS and the maximum cycle time was set to $3 \mathrm{~s}$. In charge state screening, singly assigned and 
unassigned charge states, and charge states higher than seven were rejected. Precursor masses previously selected for MS/MS measurement were excluded from further selection for $20 \mathrm{~s}$, and the exclusion window was set at $10 \mathrm{ppm}$. The samples were acquired using internal lock mass calibration on $\mathrm{m} / \mathrm{z} 371.1012$ and 445.1200. The data obtained from the mass spectrometry proteomics were handled using the local laboratory information management system (LIMS) ${ }^{64}$.

\section{Protein identification and label free protein quantification (LFQ)}

The acquired raw MS data were processed by MaxQuant (version 1.6.2.3), followed by protein identification using the integrated Andromeda search engine ${ }^{65}$. Spectra were searched against a Swissprot Mus musculus reference proteome (taxonomy 10090, version from 2019-07-09), concatenated to its reversed decoyed fasta database and common protein contaminants. Carbamidomethylation of cysteine was treated as a fixed modification, while methionine oxidation and $\mathrm{N}$-terminal protein acetylation treated as a variable. Trypsin/P was set for enzyme specificity, allowing a minimal peptide length of 7 amino acids and a maximum of two missed-cleavages. MaxQuant Orbitrap default search settings were

used. Peptides with FDR $<0.01$ and proteins with FDR $<0.05$ were processed for further steps. Label free quantification was carried out by applying a 2 minutes window for match between runs. LFQ intensity results from MaxQuant were used for a hierarchical clustering across all samples implemented in Perseus software ${ }^{66}$.

\section{Two-group comparison analysis in proteomics}

Each sample file was kept separate in the experimental design to acquire individual quantitative values in the MaxQuant experimental design template. Fold changes of proteins were calculated based on intensity values reported in the proteinGroups.txt file. Filtration for proteins with 2 or more peptides allowing a maximum of 4 missing values, normalization with a modified robust $z$-score transformation and the t-test with pooled variance to compute $p$ values were implemented using the functions in $R$ package SRMService ${ }^{67}$. If all measurements of a protein are missing in one of the conditions, a pseudo fold change was calculated replacing the missing group average by the mean of $10 \%$ smallest protein intensities in that condition. Proteins showing variable expression with adjusted $p$-value < 0.05 and two-fold change were considered to be differentially expressed between the conditions.

\section{Bulk RNA-seq / proteomics correlation analysis}

An integrated dataset was created with differentially expressed genes overlapping with differentially expressed proteins based on their common Ensembl identifiers. Pearson correlation coefficient was computed between differentially expressed genes and proteins using their log2 fold change values.

\section{Enrichment analysis for functional annotation}

For bulk RNA-seq data, the pathway enrichment analysis was carried out using Gene Set Enrichment analysis (GSEA) based on genes ranked in order of log2FC and the functional WikiPathways (www.wikipathways.org) database via WEB-based GEne SeT AnaLysis Toolkit (WebGestalt) ${ }^{68}$. Only significant $($ FDR $<0.05)$ pathway categories with ten to 500 genes were considered for enrichment analyses. For proteomics and integration between bulk RNA-Seq and proteomics datasets, enrichment analysis was performed based on significant DEGs / DEPs (|Log2FC $\mid>1$, adj.P-value < 0.05) in Metacore (https://clarivate.com/products/metacore/, Clarivate Analytics, London, UK). Only significant 
(FDR < 0.05) pre-built process networks were presented. For bulk RNA-seq data comparison between Pax7-nGFP ${ }^{+}$iMPCs and Pax7-nGFP ${ }^{+}$myoblasts, overrepresentation analysis (ORA) was performed using the $\mathrm{R}$ package goseq $\mathrm{v} 1.42^{69}$.

\section{ATAC-Seq}

Libraries for ATAC-Seq were constructed using Omni-ATAC protocol as previously reported ${ }^{70}$. Briefly, 50,000 - 60,000 cells were lysed on ice by cold lysis buffer containing $0.1 \%$ NP-40 (Sigma-Aldrich, 98379), 0.1\% Tween-20 (Sigma-Aldrich, P3416), 0.01\% Digitonin (Promega, G9441), $10 \mathrm{mM}$ Tris- $\mathrm{HCl}$ (pH 7.5), $10 \mathrm{mM} \mathrm{NaCl}, 3 \mathrm{mM} \mathrm{MgCl} 2$ in Nuclease-free water. Nuclei were collected by centrifugation and subjected then to transposition reaction in 1x Tagment DNA buffer containing 5\% Tn5 Transposase (Illumina, 20034197), 0.1\% Tween$20,0.01 \%$ Digitonin at $37^{\circ} \mathrm{C}$ for 30 mins on thermomixer, followed by DNA purification using MinElute PCR purification kit (Qiagen, 28004). To identify samples, index PCR amplification was performed by mixing $10 \mu \mathrm{L}$ of transposed DNA with $10 \mu \mathrm{L}$ nuclease-free water, $25 \mu \mathrm{L}$ NEBNext High-Fidelity 2X PCR Master Mix, 2.5uL of each $25 \mu \mathrm{M}$ Ad.1 and Ad.2, as previously published ${ }^{71}$. Total PCR cycle was determined according to the amplification graph after the first 12 cycles. To remove primer dimers and larger fragment, double-sided bead purification was carried out using AMPure XP (Beckman Coulter, A63881). Library quality was validated using TapeStation (Agilent Technologies) and sequenced on HiSeq-2500 (Illumina, Inc, California, USA) with paired end of $70 \mathrm{bp}$. All libraries had more than 50 million reads.

\section{ATAC-seq analysis}

ATAC sequencing was performed on a HiSeq2500 instrument at FGCZ. After initial quality control (adapter and low-quality base trimming) using fastp $v 0.20^{72}$, raw sequencing reads were mapped against the reference mouse genome assembly GRCm38.p6 using Bowtie2 v2.4. ${ }^{73}$. PCR duplicates were removed using the MarkDuplicates tool from Picard (https://broadinstitute.github.io/picard/). Peak calling was performed using MACS2 ${ }^{74}$ (3) with -nomodel -f BAMPE -gsize mm -keep-dup all -extsize 200 options. Called peaks were annotated using the $\mathrm{R}$ package ChIPseeker ${ }^{75}$. For each sample, fragment count matrix was generated using the $R$ package chromVAR ${ }^{76}$ based on the promoter region, defined as -1 to $1 \mathrm{~kb}$ around the transcription start site. The $\mathrm{R}$ package edgeR $v 3.28^{60}$ was used to perform differential expression analysis between different conditions using the fragment count matrices. Genes showing variable expression with $p$-value $<0.01$ and one-fold change were considered to have an open chromatin in the defined promoter region.

\section{Bulk RNA / ATAC-seq correlation analysis}

An integrated data set was created with differentially expressed genes overlapping with the genes with open chromatins in the promoter region based on their common Ensembl identifiers. Pearson correlation coefficient was calculated for the overlapping genes based on their log2 fold change values.

\section{Single cell RNA-Sequencing (scRNA-Seq)}

An early passage iMPC clone was trypsinized and filtered using a $40 \mu \mathrm{m}$ cell strainer (VWR, 734-0002) to filter out debris and fragments of myofibers. Filtered iMPCs were washed with PBS and the number of cells was counted manually using a hemocytometer with Trypan blue (Sigma-Aldrich, T8154) staining. Next, the cell pellet was re-suspended in PBS at a concentration of 1000 cells $/ \mu \mathrm{L}$ and immediately used for $10 x$ single cell library construction. 10x library was built using Single cell 3' reagent kit v3 (10xGenomics, Pleasanton, CA) 
according to the manufacturer's protocol. Briefly, cells were loaded in chromium chip B targeting $\sim 10,000$ recovered cells. Generated GEMs were cleaned, and cDNA was amplified by PCR, followed by cDNA fragmentation, end repair, A-tailing, adaptor ligation, index PCR, and double sized selection. The library was sequenced on a full SP flowcell of Novaseq 6000 (Illumina, Inc, California, USA) which allows to obtain 560 million reads for around 10,000 cells (over 40,000 reads per cell).

\section{scRNA-Seq data analysis}

CellRanger v4.0.0 pipeline was used for demultiplexing the sample, mapping raw reads to the mouse reference genome (build GRCm38.p6) and generating feature-barcode count matrix $^{77}$. The count matrix was further analyzed using Seurat v3.2.3 pipeline ${ }^{26,78}$. Droplets with unique feature counts $<250$ and $>7,200$ and mitochondrial gene counts $>5 \%$ were discarded for quality control. The filtered data was globally scaled via log normalization. The scaled data was dimensionally reduced via principal component analysis (PCA) using 2,000 highly variable genes. Cells were clustered based on first 20 principal components (PCs) using the Louvain algorithm ${ }^{79}$ with a resolution of 0.5 . Uniform manifold approximation and projection (UMAP) ${ }^{80}$ method was applied using the same PCs to visualize the clustered cells in low-dimensional place. Cluster biomarkers were identified using the Wilcoxon rank-sum test (adjusted $p$-value $<0.05$ ).

\section{Bulk RNA-Seq / scRNA-Seq correlation analysis}

To generate pseudo bulk data from scRNA-seq at the sample level, raw counts were aggregated across clusters of similar cell types (fibroblasts, progenitors). Pearson correlation coefficient was computed between the overlapping genes expressed in bulk and pseudo bulk RNA-seq data based on their log2 normalized expression values.

\section{Single cell trajectory analysis of scRNA-Seq dataset}

Unsupervised single cell trajectory analysis was performed using Monocle $3^{81,82}$. The cells were ordered in pseudotime along the learned trajectory with cluster 1 being the root. Due to the presence of a strong feature outside the focus of interest which might influence unsupervised analysis, we performed semi-supervised single cell trajectory analysis using Monocle $2^{27,81}$. Six genes were defined as markers namely Ccnb1 for cycling progenitors, Myog for committed progenitors, Myog and Tnnt2 for myocytes, Pdgfrb and Fbln2 for connective tissue / fibroblast cells and $\mathrm{Pax} 7$ for $\mathrm{Pax} 7^{+}$progenitors. A set of differential genes was selected, which co-varies with these markers. Cells were ordered using the top 1,000 differentially expressed genes based on their adjusted p-values. Each cell was assigned a pseudotime value to capture its progress during the biological process. Branch expression analysis modeling ${ }^{83}$ was performed to find the branch-dependent genes that could identify the mechanism underlying the cell fate decisions. Branch-dependent genes categorized by each cluster were annotated with GO term using the online tool DAVID v. $6.8^{84,85}$

\section{Statistical analysis}

We utilized 3 cell lines per group that were isolated from different mice for bulk RNA-Seq and other experiments including iMPC generation and Notch inhibition, whereas 4 cell lines for LC-MS were used. We utilized the software Prism to perform statistical analysis for gene expression results such as qRT-PCR data as indicated in the figure legends. Statistical analysis for multi-omics datasets was carried out as described in each respective section.

\section{References:}


1 Wosczyna, M. N. \& Rando, T. A. A Muscle Stem Cell Support Group: Coordinated Cellular Responses in Muscle Regeneration. Dev Cell 46, 135-143, doi:10.1016/j.devcel.2018.06.018 (2018).

2 Comai, G. \& Tajbakhsh, S. Molecular and cellular regulation of skeletal myogenesis. Curr Top Dev Biol 110, 1-73, doi:10.1016/B978-0-12-405943-6.00001-4 (2014).

3 Yin, H., Price, F. \& Rudnicki, M. A. Satellite cells and the muscle stem cell niche. Physiol Rev 93, 23-67, doi:10.1152/physrev.00043.2011 (2013).

4 Seale, P. et al. Pax7 is required for the specification of myogenic satellite cells. Cell 102, 777786 (2000).

5 Dumont, N. A., Bentzinger, C. F., Sincennes, M. C. \& Rudnicki, M. A. Satellite Cells and Skeletal Muscle Regeneration. Compr Physiol 5, 1027-1059, doi:10.1002/cphy.c140068 (2015). Montarras, D. et al. Direct isolation of satellite cells for skeletal muscle regeneration. Science 309, 2064-2067, doi:10.1126/science.1114758 (2005). Domenig, S. A., Palmer, A. S. \& Bar-Nur, O. in Organ Tissue Engineering Ch. Chapter 19-1, $1-62$ (2020).

8 Judson, R. N. \& Rossi, F. M. V. Towards stem cell therapies for skeletal muscle repair. NPJ Regen Med 5, 10, doi:10.1038/s41536-020-0094-3 (2020).

9 Xu, J., Du, Y. \& Deng, H. Direct lineage reprogramming: strategies, mechanisms, and applications. Cell Stem Cell 16, 119-134, doi:10.1016/j.stem.2015.01.013 (2015).

10 Davis, R. L., Weintraub, H. \& Lassar, A. B. Expression of a single transfected cDNA converts fibroblasts to myoblasts. Cell 51, 987-1000 (1987).

11 leda, M. et al. Direct reprogramming of fibroblasts into functional cardiomyocytes by defined factors. Cell 142, 375-386, doi:10.1016/j.cell.2010.07.002 (2010).

12 Vierbuchen, T. et al. Direct conversion of fibroblasts to functional neurons by defined factors. Nature 463, 1035-1041, doi:10.1038/nature08797 (2010).

13 Huang, P. et al. Induction of functional hepatocyte-like cells from mouse fibroblasts by defined factors. Nature 475, 386-389, doi:10.1038/nature10116 (2011).

14 Sekiya, S. \& Suzuki, A. Direct conversion of mouse fibroblasts to hepatocyte-like cells by defined factors. Nature 475, 390-393, doi:10.1038/nature10263 (2011).

15 Lalit, P. A. et al. Lineage Reprogramming of Fibroblasts into Proliferative Induced Cardiac Progenitor Cells by Defined Factors. Cell Stem Cell 18, 354-367, doi:10.1016/j.stem.2015.12.001 (2016).

16 Lujan, E., Chanda, S., Ahlenius, H., Sudhof, T. C. \& Wernig, M. Direct conversion of mouse fibroblasts to self-renewing, tripotent neural precursor cells. Proc Natl Acad Sci U S A 109, 2527-2532, doi:10.1073/pnas.1121003109 (2012).

17 Ring, K. L. et al. Direct reprogramming of mouse and human fibroblasts into multipotent neural stem cells with a single factor. Cell Stem Cell 11, 100-109, doi:10.1016/j.stem.2012.05.018 (2012).

18 Ito, N., Kii, I., Shimizu, N., Tanaka, H. \& Takeda, S. Direct reprogramming of fibroblasts into skeletal muscle progenitor cells by transcription factors enriched in undifferentiated subpopulation of satellite cells. Sci Rep 7, 8097, doi:10.1038/s41598-017-08232-2 (2017).

19 Sato, T. et al. Core Transcription Factors Promote Induction of PAX3-Positive Skeletal Muscle Stem Cells. Stem Cell Reports 13, 352-365, doi:10.1016/j.stemcr.2019.06.006 (2019).

20 Bar-Nur, O. et al. Direct Reprogramming of Mouse Fibroblasts into Functional Skeletal Muscle Progenitors. Stem Cell Reports 10, 1505-1521, doi:10.1016/j.stemcr.2018.04.009 (2018).

21 Cacchiarelli, D. et al. Aligning Single-Cell Developmental and Reprogramming Trajectories Identifies Molecular Determinants of Myogenic Reprogramming Outcome. Cell Syst 7, 258268 e253, doi:10.1016/j.cels.2018.07.006 (2018).

22 Dall'Agnese, A. et al. Transcription Factor-Directed Re-wiring of Chromatin Architecture for Somatic Cell Nuclear Reprogramming toward trans-Differentiation. Mol Cell 76, 453-472 e458, doi:10.1016/j.molcel.2019.07.036 (2019).

23 Cao, Y. et al. Genome-wide MyoD binding in skeletal muscle cells: a potential for broad cellular reprogramming. Dev Cell 18, 662-674, doi:10.1016/j.devcel.2010.02.014 (2010).

24 Sambasivan, R. et al. Distinct regulatory cascades govern extraocular and pharyngeal arch muscle progenitor cell fates. Dev Cell 16, 810-821, doi:10.1016/j.devcel.2009.05.008 (2009). Polo, J. M. et al. A molecular roadmap of reprogramming somatic cells into iPS cells. Cell 151, 1617-1632, doi:10.1016/j.cell.2012.11.039 (2012). 

transcriptomic data across different conditions, technologies, and species. Nature Biotechnology 36, 411-420, doi:10.1038/nbt.4096 (2018).

27 Qiu, X. et al. Reversed graph embedding resolves complex single-cell trajectories. Nature Methods 14, 979-982, doi:10.1038/nmeth.4402 (2017).

28 Zhang, L. et al. Dlk1 regulates quiescence in calcitonin receptor-mutant muscle stem cells. Stem Cells 39, 306-317, doi:10.1002/stem.3312 (2021).

29 Almada, A. E. et al. FOS licenses early events in stem cell activation driving skeletal muscle regeneration. Cell Rep 34, 108656, doi:10.1016/j.celrep.2020.108656 (2021).

30 Yue, L., Wan, R., Luan, S., Zeng, W. \& Cheung, T. H. Dek Modulates Global Intron Retention during Muscle Stem Cells Quiescence Exit. Dev Cell 53, 661-676 e666, doi:10.1016/j.devcel.2020.05.006 (2020).

31 Leung, C. et al. Lgr5 Marks Adult Progenitor Cells Contributing to Skeletal Muscle Regeneration and Sarcoma Formation. Cell Rep 33, 108535, doi:10.1016/j.celrep.2020.108535 (2020).

32 Baghdadi, M. B. et al. Reciprocal signalling by Notch-Collagen V-CALCR retains muscle stem cells in their niche. Nature 557, 714-718, doi:10.1038/s41586-018-0144-9 (2018).

33 Zhang, L. et al. The CalcR-PKA-Yap1 Axis Is Critical for Maintaining Quiescence in Muscle Stem Cells. Cell Rep 29, 2154-2163 e2155, doi:10.1016/j.celrep.2019.10.057 (2019).

34 Kawabe, Y., Wang, Y. X., McKinnell, I. W., Bedford, M. T. \& Rudnicki, M. A. Carm1 regulates Pax7 transcriptional activity through MLL1/2 recruitment during asymmetric satellite stem cell divisions. Cell Stem Cell 11, 333-345, doi:10.1016/j.stem.2012.07.001 (2012).

35 Knopp, P., Figeac, N., Fortier, M., Moyle, L. \& Zammit, P. S. Pitx genes are redeployed in adult myogenesis where they can act to promote myogenic differentiation in muscle satellite cells. Dev Biol 377, 293-304, doi:10.1016/j.ydbio.2013.02.011 (2013).

36 Schmidt, K., Glaser, G., Wernig, A., Wegner, M. \& Rosorius, O. Sox8 is a specific marker for muscle satellite cells and inhibits myogenesis. J Biol Chem 278, 29769-29775, doi:10.1074/jbc.M301539200 (2003).

37 Bjornson, C. R. et al. Notch signaling is necessary to maintain quiescence in adult muscle stem cells. Stem Cells 30, 232-242, doi:10.1002/stem.773 (2012).

38 Mourikis, P. et al. A critical requirement for notch signaling in maintenance of the quiescent skeletal muscle stem cell state. Stem Cells 30, 243-252, doi:10.1002/stem.775 (2012).

39 Brohl, D. et al. Colonization of the satellite cell niche by skeletal muscle progenitor cells depends on Notch signals. Dev Cell 23, 469-481, doi:10.1016/j.devcel.2012.07.014 (2012).

40 Evin, G., Sernee, M. F. \& Masters, C. L. Inhibition of $y$-Secretase as a Therapeutic Intervention for Alzheimer's Disease. CNS Drugs 20, 351-372, doi:10.2165/00023210200620050-00002 (2006).

41 Eliazer, S. et al. Wnt4 from the Niche Controls the Mechano-Properties and Quiescent State of Muscle Stem Cells. Cell Stem Cell 25, 654-665 e654, doi:10.1016/j.stem.2019.08.007 (2019).

42 Sampath, S. C. et al. Induction of muscle stem cell quiescence by the secreted niche factor Oncostatin M. Nat Commun 9, 1531, doi:10.1038/s41467-018-03876-8 (2018).

43 Latroche, C. et al. Coupling between Myogenesis and Angiogenesis during Skeletal Muscle Regeneration Is Stimulated by Restorative Macrophages. Stem Cell Reports 9, 2018-2033, doi:10.1016/j.stemcr.2017.10.027 (2017).

44 Lukjanenko, L. et al. Aging Disrupts Muscle Stem Cell Function by Impairing Matricellular WISP1 Secretion from Fibro-Adipogenic Progenitors. Cell Stem Cell 24, 433-446 e437, doi:10.1016/j.stem.2018.12.014 (2019).

45 Rando, T. A. \& Blau, H. M. Primary mouse myoblast purification, characterization, and transplantation for cell-mediated gene therapy. J Cell Biol 125, 1275-1287, doi:10.1083/jcb.125.6.1275 (1994).

46 Mourikis, P. \& Tajbakhsh, S. Distinct contextual roles for Notch signalling in skeletal muscle stem cells. BMC Dev Biol 14, 2, doi:10.1186/1471-213X-14-2 (2014).

47 Low, S., Barnes, J. L., Zammit, P. S. \& Beauchamp, J. R. Delta-Like 4 Activates Notch 3 to Regulate Self-Renewal in Skeletal Muscle Stem Cells. Stem Cells 36, 458-466, doi:10.1002/stem.2757 (2018). 
Verma, M. et al. Muscle Satellite Cell Cross-Talk with a Vascular Niche Maintains Quiescence via VEGF and Notch Signaling. Cell Stem Cell 23, 530-543 e539,

doi:10.1016/j.stem.2018.09.007 (2018).

49 Sakai, $H$. et al. Notch ligands regulate the muscle stem-like state ex vivo but are not sufficient for retaining regenerative capacity. PLoS One 12, e0177516, doi:10.1371/journal.pone.0177516 (2017).

50 Gerli, M. F. M. et al. Combined Notch and PDGF Signaling Enhances Migration and Expression of Stem Cell Markers while Inducing Perivascular Cell Features in Muscle Satellite Cells. Stem Cell Reports 12, 461-473, doi:10.1016/j.stemcr.2019.01.007 (2019).

$51 \mathrm{Xu}, \mathrm{C}$. et al. A zebrafish embryo culture system defines factors that promote vertebrate myogenesis across species. Cell 155, 909-921, doi:10.1016/j.cell.2013.10.023 (2013).

52 Girardi, F. et al. TGFbeta signaling curbs cell fusion and muscle regeneration. Nat Commun 12, 750, doi:10.1038/s41467-020-20289-8 (2021).

53 Melendez, J. et al. TGFbeta signalling acts as a molecular brake of myoblast fusion. Nat Commun 12, 749, doi:10.1038/s41467-020-20290-1 (2021).

54 Quarta, M. et al. An artificial niche preserves the quiescence of muscle stem cells and enhances their therapeutic efficacy. Nat Biotechnol 34, 752-759, doi:10.1038/nbt.3576 (2016).

55 Sambasivan, R. et al. Distinct Regulatory Cascades Govern Extraocular and Pharyngeal Arch Muscle Progenitor Cell Fates. Developmental Cell 16, 810-821, doi:https://doi.org/10.1016/j.devcel.2009.05.008 (2009).

56 Bosnakovski, D. et al. Prospective isolation of skeletal muscle stem cells with a Pax7 reporter. Stem Cells 26, 3194-3204, doi:10.1634/stemcells.2007-1017 (2008).

57 Hatakeyama, M. et al. SUSHI: an exquisite recipe for fully documented, reproducible and reusable NGS data analysis. BMC Bioinformatics 17, 228, doi:10.1186/s12859-016-1104-8 (2016).

58 Bolger, A. M., Lohse, M. \& Usadel, B. Trimmomatic: a flexible trimmer for Illumina sequence data. Bioinformatics 30, 2114-2120, doi:10.1093/bioinformatics/btu170 \%J Bioinformatics (2014).

59 Bray, N. L., Pimentel, H., Melsted, P. \& Pachter, L. Near-optimal probabilistic RNA-seq quantification. Nature Biotechnology 34, 525-527, doi:10.1038/nbt.3519 (2016).

60 Robinson, M. D., McCarthy, D. J. \& Smyth, G. K. edgeR: a Bioconductor package for differential expression analysis of digital gene expression data. Bioinformatics 26, 139-140, doi:10.1093/bioinformatics/btp616 \%J Bioinformatics (2009).

61 FUTSCHIK, M. E. \& CARLISLE, B. NOISE-ROBUST SOFT CLUSTERING OF GENE EXPRESSION TIME-COURSE DATA. 03, 965-988, doi:10.1142/s0219720005001375 (2005). Kumar, L. \& E Futschik, M. Mfuzz: a software package for soft clustering of microarray data. Bioinformation 2, 5-7, doi:10.6026/97320630002005 (2007).

63 Szklarczyk, D. et al. STRING v11: protein-protein association networks with increased coverage, supporting functional discovery in genome-wide experimental datasets. Nucleic Acids Res 47, D607-D613, doi:10.1093/nar/gky1131 \%J Nucleic Acids Research (2018).

64 Türker, C. et al. in Proceedings of the 13th International Conference on Extending Database Technology 717-720 (Association for Computing Machinery, Lausanne, Switzerland, 2010).

65 Cox, J. \& Mann, M. MaxQuant enables high peptide identification rates, individualized p.p.b.range mass accuracies and proteome-wide protein quantification. Nat Biotechnol 26, 13671372, doi:10.1038/nbt.1511 (2008).

66 Tyanova, S. et al. The Perseus computational platform for comprehensive analysis of (prote)omics data. Nature Methods 13, 731-740, doi:10.1038/nmeth.3901 (2016).

67 W. Wolski, J. G., C. Panse. SRMService - R-Package to Report Quantitative Mass Spectrometry Data., <http://github.com/protViz/SRMService> (2018).

68 Zhang, B., Kirov, S. \& Snoddy, J. WebGestalt: an integrated system for exploring gene sets in various biological contexts. Nucleic Acids Res 33, W741-W748, doi:10.1093/nar/gki475 (2005).

69 Young, M. D., Wakefield, M. J., Smyth, G. K. \& Oshlack, A. Gene ontology analysis for RNAseq: accounting for selection bias. Genome Biology 11, R14, doi:10.1186/gb-2010-11-2-r14 (2010).

70 Corces, M. R. et al. An improved ATAC-seq protocol reduces background and enables interrogation of frozen tissues. Nature Methods 14, 959-962, doi:10.1038/nmeth.4396 (2017). 
71 Buenrostro, J. D., Giresi, P. G., Zaba, L. C., Chang, H. Y. \& Greenleaf, W. J. Transposition of native chromatin for fast and sensitive epigenomic profiling of open chromatin, DNA-binding proteins and nucleosome position. Nature Methods 10, 1213-1218, doi:10.1038/nmeth.2688 (2013).

72 Chen, S., Zhou, Y., Chen, Y. \& Gu, J. fastp: an ultra-fast all-in-one FASTQ preprocessor. Bioinformatics 34, i884-i890, doi:10.1093/bioinformatics/bty560 \%J Bioinformatics (2018).

73 Langmead, B. \& Salzberg, S. L. Fast gapped-read alignment with Bowtie 2. Nature Methods 9 , 357-359, doi:10.1038/nmeth.1923 (2012).

74 Zhang, Y. et al. Model-based Analysis of ChIP-Seq (MACS). Genome Biology 9, R137, doi:10.1186/gb-2008-9-9-r137 (2008).

$75 \quad$ (!!! INVALID CITATION !!! \{\}).

76 Schep, A. N., Wu, B., Buenrostro, J. D. \& Greenleaf, W. J. chromVAR: inferring transcriptionfactor-associated accessibility from single-cell epigenomic data. Nature Methods 14, 975-978, doi:10.1038/nmeth.4401 (2017).

77 Zheng, G. X. Y. et al. Massively parallel digital transcriptional profiling of single cells. Nature Communications 8, 14049, doi:10.1038/ncomms14049 (2017).

78 Stuart, T. et al. Comprehensive Integration of Single-Cell Data. Cell 177, 1888-1902.e1821, doi:https://doi.org/10.1016/j.cell.2019.05.031 (2019).

79 Blondel, V. D., Guillaume, J.-L., Lambiotte, R. \& Lefebvre, E. Fast unfolding of communities in large networks. Journal of Statistical Mechanics: Theory and Experiment 2008, P10008, doi:10.1088/1742-5468/2008/10/p10008 (2008).

80 Becht, E. et al. Dimensionality reduction for visualizing single-cell data using UMAP. Nature Biotechnology 37, 38-44, doi:10.1038/nbt.4314 (2019).

81 Trapnell, C. et al. The dynamics and regulators of cell fate decisions are revealed by pseudotemporal ordering of single cells. Nature Biotechnology 32, 381-386, doi:10.1038/nbt.2859 (2014).

82 Cao, J. et al. The single-cell transcriptional landscape of mammalian organogenesis. Nature 566, 496-502, doi:10.1038/s41586-019-0969-x (2019).

83 Qiu, X. et al. Single-cell mRNA quantification and differential analysis with Census. Nature Methods 14, 309-315, doi:10.1038/nmeth.4150 (2017).

84 Huang, D. W., Sherman, B. T. \& Lempicki, R. A. Systematic and integrative analysis of large gene lists using DAVID bioinformatics resources. Nature Protocols 4, 44-57, doi:10.1038/nprot.2008.211 (2009).

85 Huang, D. W., Sherman, B. T. \& Lempicki, R. A. Bioinformatics enrichment tools: paths toward the comprehensive functional analysis of large gene lists. Nucleic Acids Res 37, 1-13, doi:10.1093/nar/gkn923\%J Nucleic Acids Research (2008).

Figure legends:

Fig. 1: A reprogrammable system to study fibroblasts conversion into myogenic cells (A) A schematic of experimental design denoting the conversion of Thy $1^{+}$Pax7-nGFP RepMEFs into multinucleated myotubes solely by MyoD overexpression or iMPCs by MyoD+F/R/C treatment. MEFs, mouse embryonic fibroblasts; Dox, Doxycycline. (B) Representative bright-field images of Thy $1^{+}$Rep-MEFs subjected to MyoD or MyoD+F/R/C treatment for the indicated days. Scale bar, $400 \mu \mathrm{m}$. (C) qRT-PCR for canonical myogenic genes. Data is shown as means $\square \pm \square$ S.D. $N=3$ cell lines per group. Statistical significance was determined by a two-tailed unpaired $t$-test $\left({ }^{*} p<0.05,{ }^{* *} p<0.01,{ }^{* * *} p<0.001\right.$, n.s $=$ nonsignificant). (D) Representative images of a stable Pax7-nGFP iMPC clone at passage 1. Scale bar, $100 \mu \mathrm{m}$. (E) Flow cytometry analysis of a Pax7-nGFP iMPC clone. (F) Representative immunofluorescence images of Pax7-nGFP iMPCs immunostained for Mki67 and Pax7. Nuclei were counterstained with 4',6-diamidino-2-phenylindole (DAPI). Scale bar, $100 \mu \mathrm{m}$. (G) Representative immunofluorescence images of Pax7-nGFP iMPCs immunostained for MyoD and MyHC. Nuclei were counterstained with DAPI. White arrowheads point to mononucleated Pax7-nGFP $P^{+}$cells that are negative for MyoD. Scale bar, 
$100 \mu \mathrm{m}$. (H) Quantification of (f). $\mathrm{N}=4$ field images that were taken from the respective iMPC line.

\section{Fig. 2: Global transcriptome dynamics during myogenic reprogramming and transdifferentiation}

(A) An experimental design for the bulk RNA-Seq analysis. (B) PCA of global RNA-Seq data using all gene read counts. $N=3$ cell lines per group. (C) Graph showing the numbers of DEGs between each indicated comparison. DEGs were calculated using $|\log 2 \mathrm{FC}|>0.5$ ( $\mathrm{p}$ value $<0.01$ ). DEGs, differentially expressed genes. (D) Gene expression dynamics for the indicated markers based on bulk RNA-Seq data during MyoD or MyoD+F/R/C conversion. Established iMPCs and primary myoblasts served as positive controls. Relative gene expression was calculated by normalizing the Reads per Kilobase Million (RPKM) values of each sample to that of parental MEFs (D0 MEFs). The data is shown as means $\square \pm \square$ S.D. $N=$ 3 cell lines per group. Statistical significance was determined by two-way ANOVA between conditions at each time point $\left({ }^{*} p<0.05,{ }^{* *} p<0.01,{ }^{* * *} p<0.001,{ }^{* * * *} p<0.0001\right)$. (E) Fuzzy clustering based on bulk RNA-Seq data for gene expression dynamics across the indicated days in MEFs subjected to MyoD or MyoD+F/R/C treatment. Expression changes are represented as log2FC for each time point vs. parental MEFs. $N=3$ cell lines per each group. The number of genes is shown together with the respective GO term annotation and representative genes in each gene set. GO term and gene annotations were defined using the STRING database. (F) Volcano plots showing DEGs between MyoD and MyoD+F/R/C conditions at the indicated days. Significant DEGs $(|\log 2 \mathrm{FC}|>0.5, \mathrm{p}$-value $<0.05)$ are shown as yellow dots. $\mathrm{N}=3$ cell lines per group. (G) $A$ heatmap of enriched pathways in MyoD+F/R/C vs. MyoD conditions at the indicated time points. Normalized enrichment score (nES) obtained via Gene Set Enrichment Analysis (GSEA) using WikiPathways database is displayed. $\mathrm{N}=3$ cell lines per group. $(\mathbf{H}) \mathrm{A}$ heatmap of relative gene expression based on bulk RNA-Seq data for each of the indicated genes at the respective time points. Each gene group is associated with the respective pathway shown in (E). Log2FC was calculated for MyoD+F/R/C vs. MyoD condition. $\mathrm{N}=3$ cell lines per group.

\section{Fig. 3: Proteome dynamics during iMPC formation}

(A) Hierarchical clustering based on total proteome data. $\mathrm{N}=4$ cell lines per group. (B) Pie charts showing quantification of DEPs between the indicated cell lines. The significance threshold was set at $|\log 2 \mathrm{FC}|>1$ and adj. $\mathrm{p}$-value $<0.05$. $\mathrm{N}=4$ cell lines per group. (C) $\mathrm{A}$ heatmap showing relative protein expression for canonical myogenic markers in the indicated cell lines. The calculated log2FC in each cell line vs. MEFs is shown. $\mathrm{N}=4$ cell lines per group. (D) Heatmaps based on proteome data displaying the top 30 upregulated proteins in the indicated cell lines vs. parental MEFs. The average expression is presented as gradient. $\mathrm{N}=4$ cell lines per group. Canonical myogenic differentiation-associated proteins are highlighted in red. (E) Pie chart showing quantification of DEPs between MEFs subjected to MyoD or MyoD+F/R/C conditions at day 10. The significance threshold was set as $|\log 2 \mathrm{FC}|>1$ and adj. $\mathrm{p}$-value $<0.05 . \mathrm{N}=4$ cell lines per group. (F) Volcano plot for DEPs between MEFs subjected to MyoD and MyoD+F/R/C conditions at day 10 of reprogramming. Significant DEPs $(|\log 2 \mathrm{FC}|>1$, adj.p-value $<0.05)$ are shown as blue and red dots. $\mathrm{N}=4$ cell lines per group. (G) A heatmap of highly expressed proteins in MEFs subjected to MyoD+F/R/C conditions vs. MyoD condition at day 10 of reprogramming shown together with their respective cluster annotation. The average expression is presented as gradient. $\mathrm{N}$ $=4$ cell lines per group. $(\mathbf{H})$ Left- A Venn diagram showing the number of statistically 
significant DEGs and DEPs identified in MyoD+F/R/C vs. MyoD conditions at day 10 of reprogramming ( $|\log 2 \mathrm{FC}|>1, p$-value $<0.05)$. Right- scatterplot showing the correlation between the transcriptome and proteome of MyoD+F/R/C vs. MyoD conditions at day 10 of reprogramming. 180 overlapped DEGs / DEPs are projected on the plot, corresponding to the Venn diagram on the left. (I) Scatterplot showing the correlated Process networks that are enriched at the mRNA and protein levels in MyoD+F/R/C vs. MyoD treated MEFs at day 10. Enrichment analysis of DEGs / DEPs ( $|\log 2 \mathrm{FC}|>1$, p-value $<0.05)$ was performed using Metacore. Upregulated (in Red) and downregulated (in Blue) Process networks are shown using - $\log 10($ FDR) and $\log 10($ FDR $)$, respectively.

\section{Fig.4: Chromatin accessibility dynamics unique to iMPC reprogramming}

(A) Correlation matrix for the indicated samples based on ATAC-Seq for global chromatin accessibility. Pearson's correlation coefficient $r$ is displayed as a color gradient. (B) Distribution of annotated ATAC-Seq peaks across the respective genomic regions. (C) Heatmap showing differential chromatin accessibility of the indicated gene promoter regions ( $\pm 1 \mathrm{~kb}$ of TSS) compared to MEFs. Log2FC is shown via a color gradient and calculated vs. MEFs. Non-significant values ( $p$-value $>0.01$ ) are shown in white. $\mathrm{N}=2$ cell lines per group. (D) Scatterplot of selected genes showing the correlation between chromatin accessibility in promoter regions ( $\pm 1 \mathrm{~kb}$ of TSS) and bulk RNA-Seq in day 2 MyoD+F/R/C vs. MyoD treated MEFs (left) and iMPCs vs. MEFs (right). The genes with $|\log 2 \mathrm{FC}|>0.5$ (left) and $|\log 2 \mathrm{FC}|>$ 2 (right) are presented with p-value $<0.01$. (E) IGV tracks of ATAC-Seq peaks and bulk RNA-Seq for the indicated genes.

Fig. 5: scRNA-Seq uncovers cell types and differentiation trajectories in iMPCs

(A) UMAP projection of the scRNA-Seq data showing 9,184 cells colored by clusters that form a stable iMPC clone. (B) UMAP projection colored by 3 main cell cycle states in an iMPC clone. (C) Dot plot showing the expression of the indicated genes in each cell cluster. The size of the dot indicates the percentage of cells expressing the indicated genes within the cluster. The color-coding scale represents the average gene expression level in all cells in a cluster. (D) UMAP projection showing all cells colored by the indicated myogenic markers based on scRNA-Seq data. (E) UMAP projection showing all cells colored by pseudotime as calculated from unsupervised single cell trajectory as reconstructed by Monocle 3. (F-G) Minimum spanning tree showing ordered cells based on semi-supervised single cell trajectory analysis reconstructed by Monocle2 and colored by either pseudotime $(F)$ or cell cluster identifiers $(G)$. The pseudotime trajectory initiates from the $F_{0}$ root and bifurcates at the branch point $\mathrm{B}_{1}$ before proceeding towards two main cell fates (denoted as $F_{1}$ and $\left.F_{3}\right)$. (H) Plot showing the kinetics of the indicated genes in various cell types as a function of pseudotime $(F)$ emanating from point $F_{0}$ and proceeding towards $F_{1}$ (Solid line, "myogenic cell fate") or to $\mathrm{B}_{2}$ (Dashed line, "connective tissue cell fate"). Dots indicate cells colored by cell identifiers. (I) Heatmap based on scRNA-Seq data for the top 50 DEGs regulated at branch point $B_{1}$ in $(F)$. The individual gene expression level initiating at point $F_{0}$ and proceeding towards a myogenic cells fate $\left(F_{0^{-}}>F_{1}\right)$ is shown on the left and connective tissue cell fate $\left(F_{0^{-}}>B_{2}\right)$ is shown on the right. Color-code gradient shows normalized gene expression level (Z-score) for each gene across all cells. The GO terms were annotated with DAVID v6.8 for each respective gene group shown using the same color coding.

Fig. 6: Molecular characterization of FACS-purified Pax7-nGFP ${ }^{+}$iMPCs and myoblasts 
(A) A schematic illustrating the strategy to FACS-purify Pax7-nGFP ${ }^{+}$cells from heterogeneous iMPCs or primary myoblasts prior to bulk RNA-Seq and proteomic analyses. (B) Heatmap based on bulk RNA-Seq data showing the expression level of skeletal muscle related genes in the indicated samples. Log2FC of respective cell types vs. MEFs is shown. $N=3$ cell lines per group. (C) Pathway enrichment analysis using GSEA between bulk iMPCs and Pax7-nGFP ${ }^{+}$iMPCs. (D) Heatmap based on bulk RNA-Seq dataset demonstrating the expression level of the indicated genes between Pax7-nGFP ${ }^{+}$iMPCs and bulk iMPCs. Each gene group is associated with the respective pathway annotation in (C). The average expression is presented as gradient. $N=3$ cell lines per group. (E) Scatter plot showing gene expression based on bulk RNA-Seq data between Pax7-nGFP ${ }^{+}$iMPCs and Pax7-nGFP ${ }^{+}$myoblasts. Log2 of normalized read counts is presented. Red and blue dots denote upregulated genes $\left(\log 2 \mathrm{FC}>1\right.$ ) in Pax7-nGFP ${ }^{+}$myoblasts or Pax7-nGFP ${ }^{+}$iMPCs, respectively. Gray dots indicate non-significant genes between the two cell types. $\mathrm{N}=3$ cell lines per group. (F) Over Representation Analysis (ORA) of the gene sets that were significantly enriched $(\log 2 \mathrm{FC}>0.5, \mathrm{p}$-value $<0.01)$ in Pax7-nGFP ${ }^{+}$iMPCs in comparison to Pax7-nGFP ${ }^{+}$myoblasts. The size indicates the number of genes involved in the annotation and the color-coding scales the fold change. (G-H) Heatmaps based on bulk RNA-Seq data of candidate genes associated with satellite cell markers $(G)$ and signaling pathways $(H)$ that are highly expressed in Pax7-nGFP ${ }^{+}$iMPCs. The average expression is presented via a gradient. $\mathrm{N}=3$ cell lines per each group. (I) A Venn diagram based on proteome data showing the total number of proteins detected in Pax7-nGFP ${ }^{+}$myoblasts and Pax7-nGFP ${ }^{+}$ iMPCs. $\mathrm{N}=4$ cell lines per group. (J) Number of DEPs in Pax7-nGFP ${ }^{+}$myoblasts vs. Pax7$n \mathrm{GFP}^{+}$iMPCs. The significance threshold was set as $|\log 2 \mathrm{FC}|>1$ and adj. $p$-value $<0.1 . \mathrm{N}=4$ cell lines per group. (K) Scatter plot based on log2 normalized protein expression between Pax7-nGFP ${ }^{+}$iMPCs and Pax7-nGFP $^{+}$myoblasts. $\mathrm{N}=4$ cell lines per group.

Fig. 7: Notch signaling is indispensable for the formation and maintenance of iMPCs

(A) Expression dynamics based on bulk RNA-Seq data for the indicated Notch pathway associated genes during MyoD or MyoD+F/R/C reprogramming course. Established iMPCs and primary myoblasts served as positive controls. Relative gene expression was calculated by normalizing the RPKM values of each sample to that of parental MEFs (D0 MEFs). $N=3$ cell lines per group. Statistical significance was determined by two-way ANOVA between the conditions at each time point $\left({ }^{*} p<0.05,{ }^{* *} p<0.01,{ }^{* * *} p<0.001,{ }^{* * * *} p<0.0001\right)$. (B) Representative bright-field images of MEFs subjected to MyoD, MyoD+F/R/C and MyoD+F/R/C+DAPT treatment at day 10 of reprogramming. Scale bar, $400 \mu \mathrm{m}$. (C) Representative immunofluorescence images of MEFs subjected to the listed conditions at day 10 of reprogramming and stained for Pax7, MyHC and DAPI. Scale bar, $100 \mu \mathrm{m}$. (D) qRT-PCR analysis for the indicated genes and conditions. Relative gene expression is shown as means $\square \pm \square$ S.D. $N=3$ cell lines per each group. Statistical significance was determined by one-way ANOVA $\left({ }^{*} p<0.05,{ }^{* *} p<0.01,{ }^{* * *} p<0.001,{ }^{* * *} p<0.0001\right.$, n.s $=$ nonsignificant). (E) Representative bright-field images of iMPCs (P6) treated or non-treated with DAPT for 5 consecutive days. Scale bar, $400 \mu \mathrm{m}$. (F) Quantification of the total number of cells in iMPCs treated or non-treated with DAPT for 5 consecutive days. Statistical significance was determined by a two-tailed unpaired $t$-test $\left({ }^{* * *} p<0.0001\right) . \quad N=2$ experimental repeats of 3 cell lines per group. (G) Flow cytometry analysis for Pax7-nGFP iMPCs treated or non-treated with DAPT for 5 days. (H) Representative immunofluorescence images for Pax7, MyHC and DAPI in iMPCs treated with and without DAPT for 5 consecutive days. Scale bar, $100 \mu \mathrm{m}$. (I) qRT-PCR for selective genes in DAPT-treated iMPCs for 5 
consecutive days. The data is shown as means $\square \pm \square$ S.D. $N=3$ cell lines per group. Statistical significance was determined by one-way ANOVA $\left({ }^{* *} p<0.01\right.$, ${ }^{* * *} p<0.001$, n.s $=$ non-significant). (J) Violin plots showing the average expression of the indicated genes in the respective clusters based on scRNA-Seq data from Fig 5. Note presence of canonical Notch-related markers in Pax $7^{+}$iMPCs (clusters $1,2,4$ ) as well as typical Notch ligands and inhibitors in their differentiated progeny (clusters 5, 6). 
Figure 1 bioRxiv preprint doi: https://doi.org/10.1101/2021.08.20.457151; this version posted August 21, 2021. The copyright holder for this preprint Fure (which was not certified by peer review) is the author/funder, who has granted bioRxiv a license to display the preprint in perpetuity. It is made A

$\begin{array}{ll}\text { Pax7-nGFP mouse } & \text { +LV-tetO-MyoD/PGK-Puromycin } \\ & \text { +LV-EF1a-rtTA3/PGK-Neomycin }\end{array}$
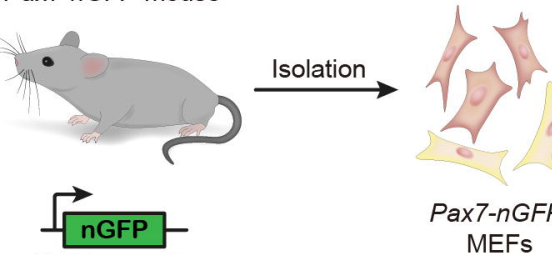

Pax7 promoter
Pax7-nGFP MEFs

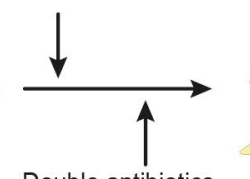

Double antibiotics selection

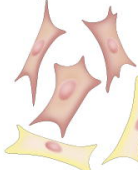

Reprogrammable Pax7-nGFP MEFs

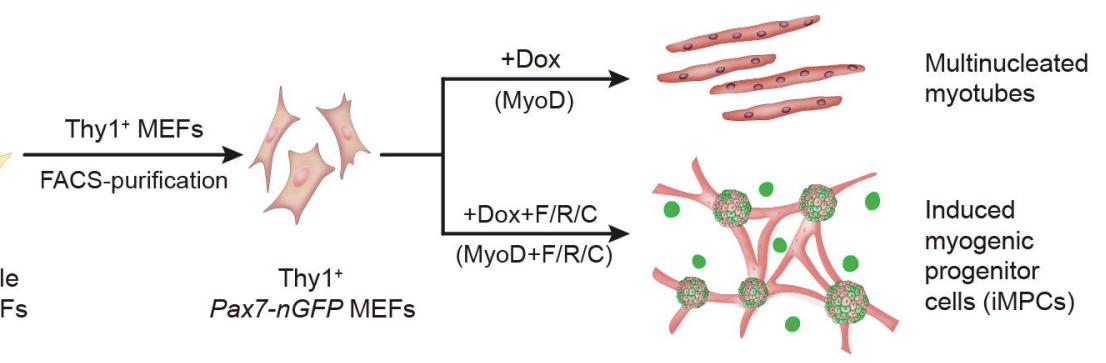

F- Forskolin (cAMP agonist)

R- RepSox (TGF- $\beta$ receptor inhibitor) C- CHIR99021 (GSK3- $\beta$ inhibitor)
B

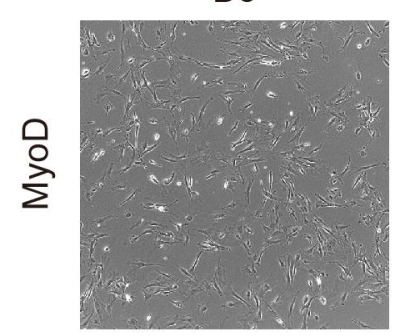

$\frac{0}{\frac{0}{\alpha}}$

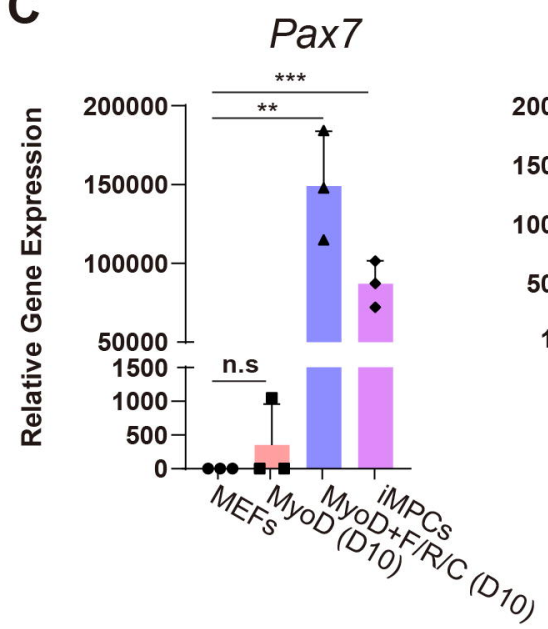

$\mathbf{F}$

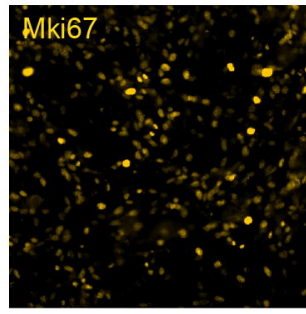

Mki67

Mikik
D2
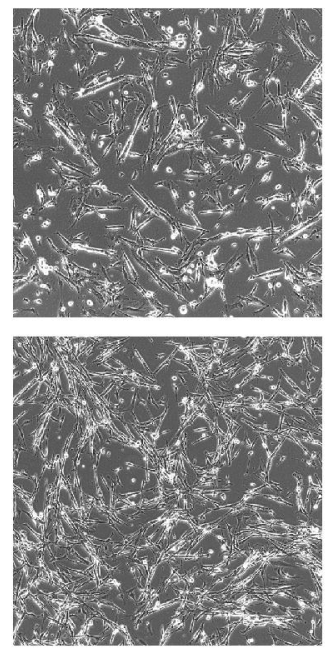

D4
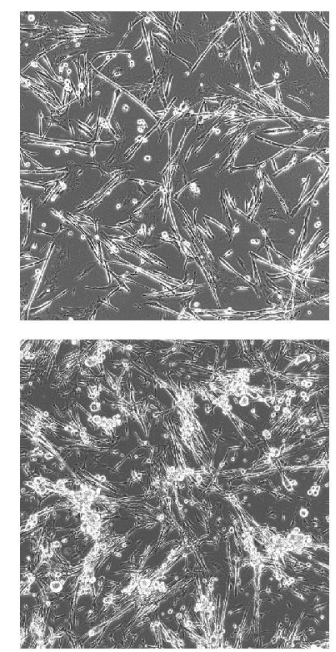

D6
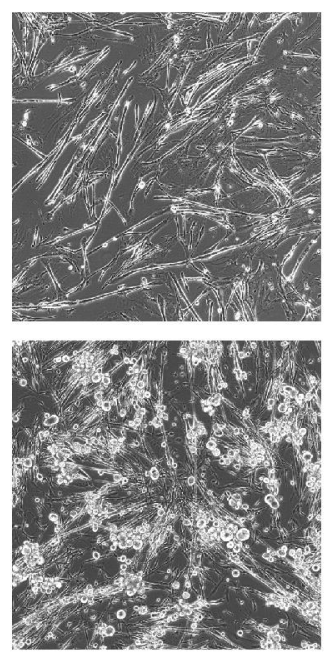

Myog

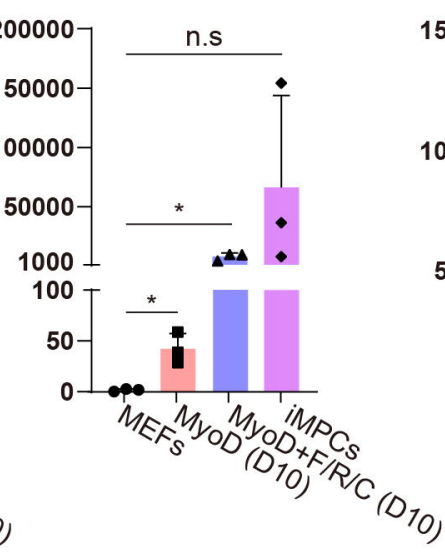

G
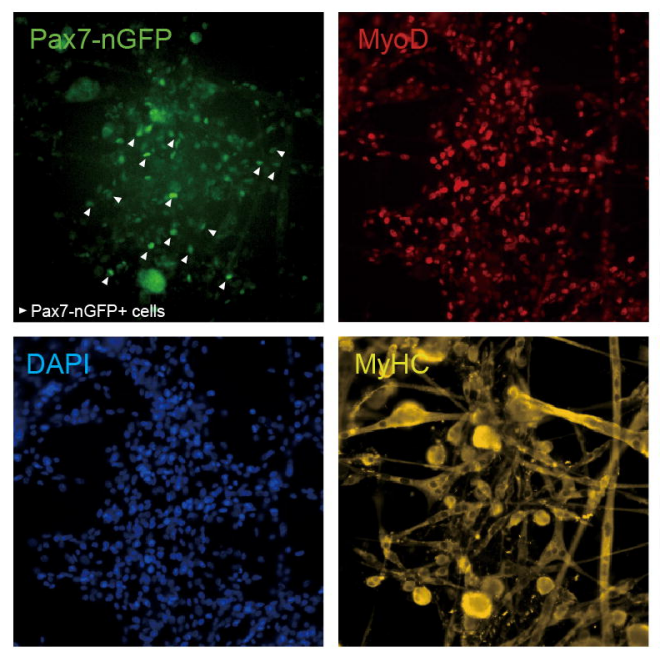

D8
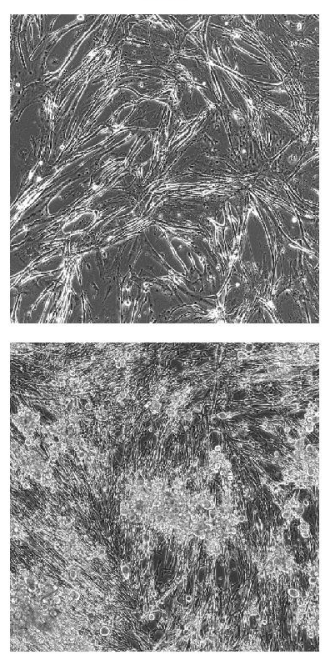

D10
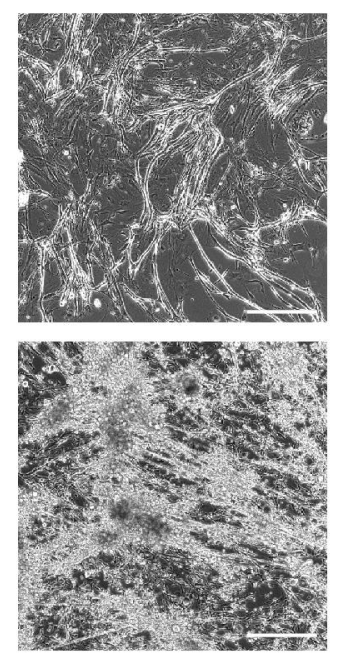

D
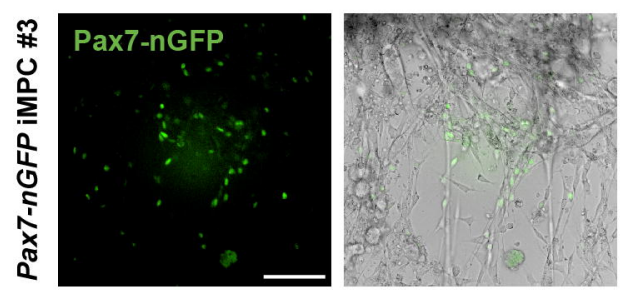

E

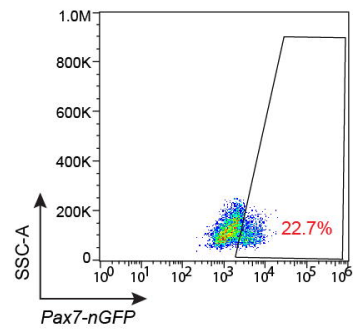

H
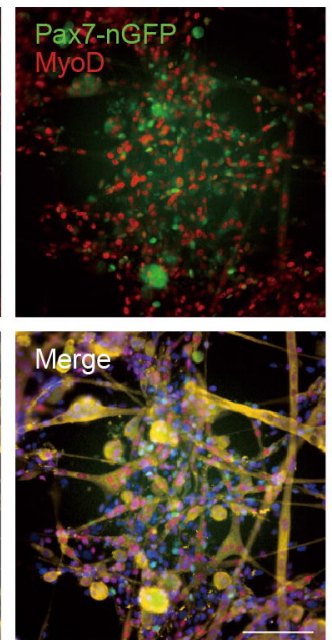

$P a x 7-n G F P$ iMPCs \#3

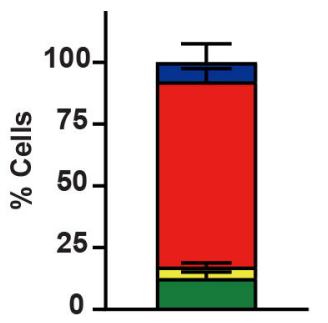

口 Pax7-/ MyoD-

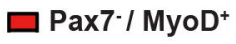

$\square \mathrm{Pax}^{+} / \mathrm{MyoD}^{+}$

$\square$ Pax7 $^{+} /$MyoD- 
Figure 2 bioRxiv preprint doi: https://doi.org/10.1101/2021.08.20.457151; this version posted August 21, 2021. The copyright holder for this preprint A
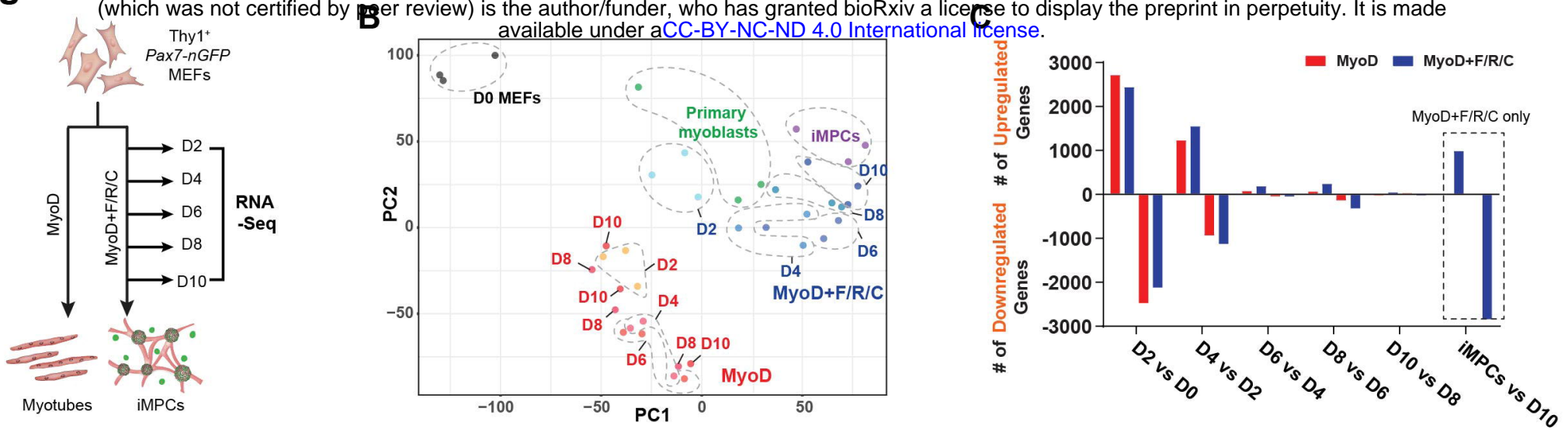

\section{D $\rightarrow$ MyoD $\rightarrow$ MyoD+F/R/C $₫$ iMPCs $\rightarrow$ Primary myoblasts}

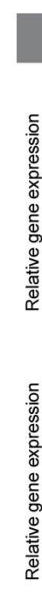
Satellite cell markers Committed progenitors

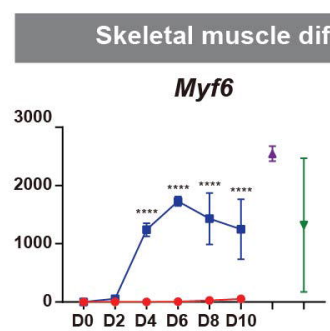

ferentiation markers
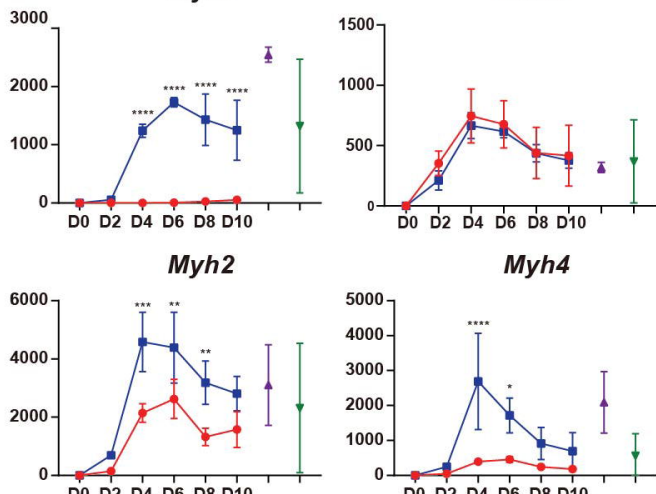

D0 D2 D4 D6 D8 D10
Tnni1

Myh4

Fibroblast markers

Thy1

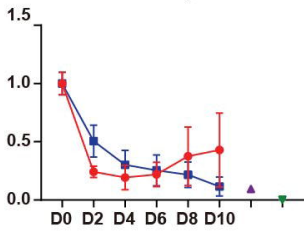

Fbln2

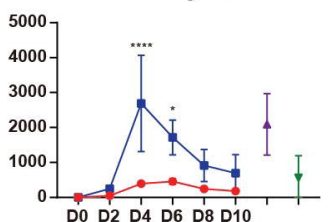

E

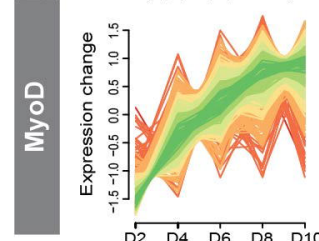

$\begin{array}{lllll}\text { D2 } & \text { D4 } & \text { D6 } & \text { D8 } & \text { D10 }\end{array}$ GS1 ( $n=117)$ Cellular metablic process

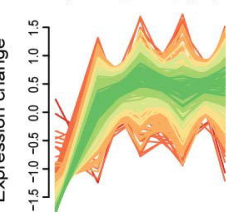

\begin{tabular}{lllll}
\hline$D_{2}$ & $D_{4}^{\prime}$ & $D^{\prime} 6$ & $D^{\prime} 8$ & D10
\end{tabular}
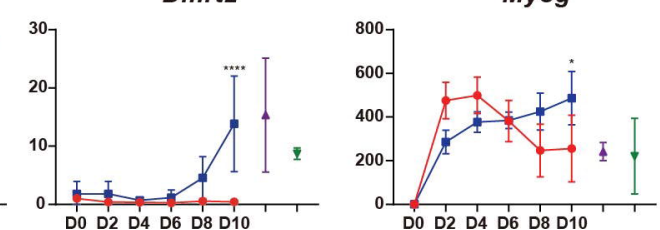

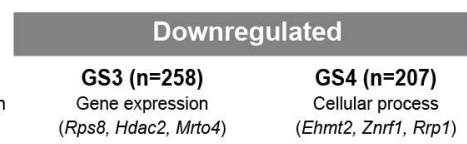

GS2 ( $n=266$ )

(Cdk2, Mcm2, Pold2)

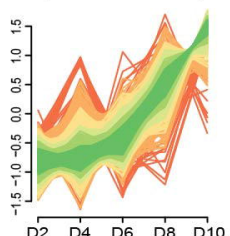

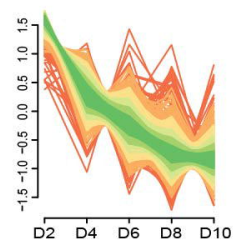
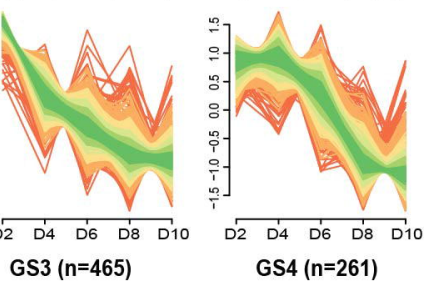

\section{GS4 ( $n=261)$}

Protein localization

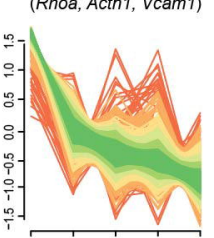

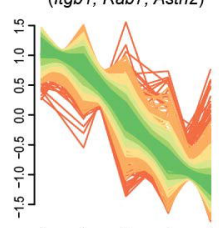

Transiently downregulated

GS5 ( $n=250) \quad$ GS6 $(n=61)$ $\begin{gathered}\text { Cellular metabolic process } \\ \text { (Mcm3, Mef2a, Acs/4) }\end{gathered}$
(Canx, Ckap4, Gsr)
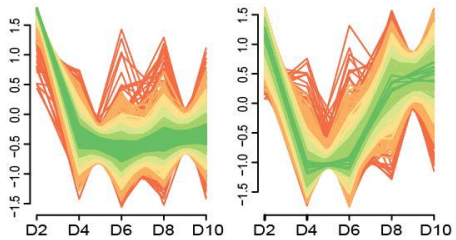

\section{GS5 ( $n=105)$}

Cellular process

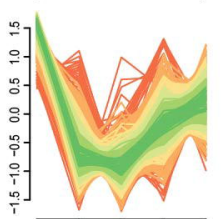

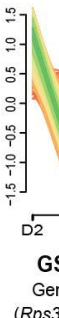

(Rps3, Nudt21, Rcc2)

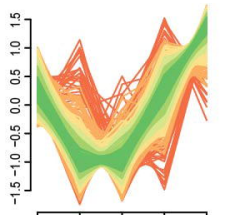

$\begin{array}{cc}\text { Transiently upregulated } \\ \text { GS7 ( } \mathbf{n = 1 7 2 )} & \text { GS8 }(\mathbf{n = 1 3 3 )} \\ \text { Myofibril assembly } & \text { Muscle contraction } \\ \text { (Akt1, Actc1, Casq2) } & \text { (Obscn, Neb, Tnnc2) }\end{array}$
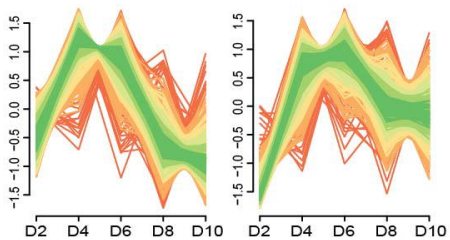

GS7 ( $n=206)$

Intracellular transport
(Syvn1, Klc1, Rab 10$)$

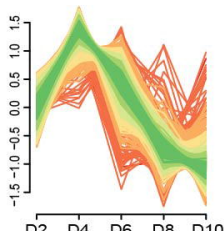

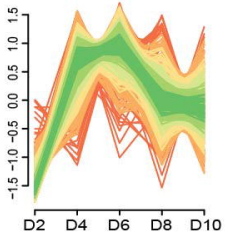

GS8 ( $n=203)$

sce structure development

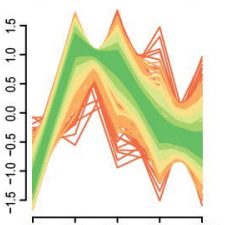

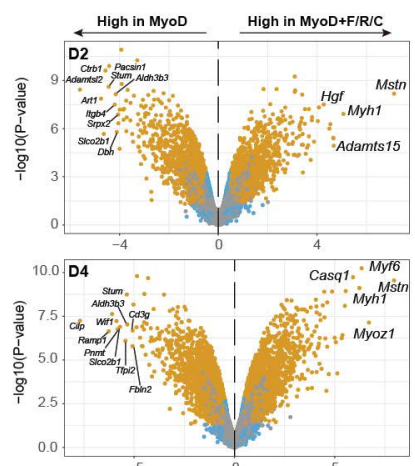

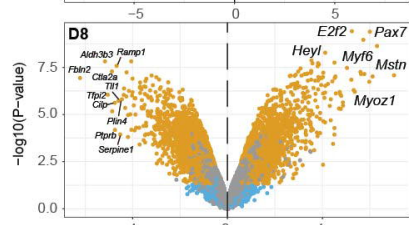

G Pathway enrichment analysis (MyoD+F/R/C vs. MyoD)

\begin{tabular}{|c|c|c|c|}
\hline $\mathrm{D} 2 \mathrm{D}$ & $24 \quad D 6$ & D8 D10 & \\
\hline & & & Notch Signaling Pathway \\
\hline & & & Hedgehog Signaling Pathway \\
\hline & & & Factors and pathways affecting insulin-like growth factor (IGF1)-Akt signaling \\
\hline & & & G1 to $S$ cell cycle control \\
\hline & & & DNA Replication \\
\hline & & & Eukaryotic Transcription Initiation \\
\hline & & & mRNA processing \\
\hline & & & Cytoplasmic Ribosomal Proteins \\
\hline & & & Oxidative phosphorylation \\
\hline & & & TCA Cycle \\
\hline & & & Electron Transport Chain \\
\hline & & & Cholesterol Biosynthesis \\
\hline & & & Cholesterol metabolism (includes both Bloch and Kandutsch-Russell pathways) \\
\hline & & & Glycolysis and Gluconeogenesis \\
\hline & & & One Carbon Metabolism \\
\hline & & & Glutathione and one carbon metabolism \\
\hline & & & TGF Beta Signaling Pathway \\
\hline & & & Focal Adhesion \\
\hline
\end{tabular}

$\mathbf{H}$

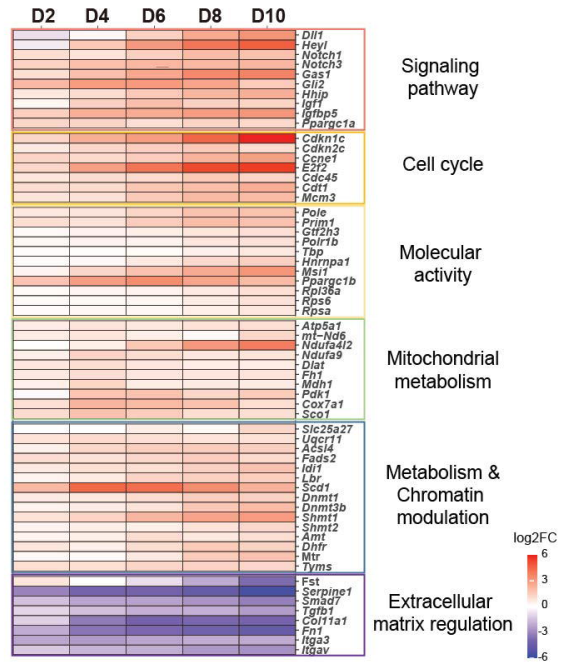


Figure 3 bioRxiv preprint doi: https://doi.org/10.1101/2021.08.20.457151; this version posted August 21, 2021. The copyright holder for this preprint

A

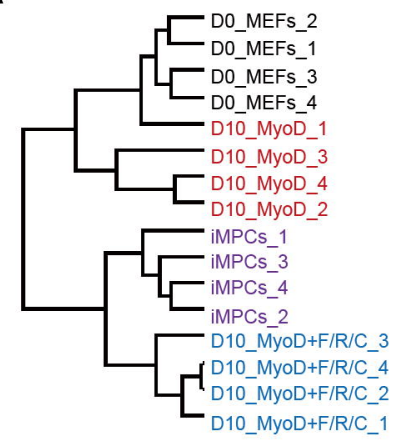

B

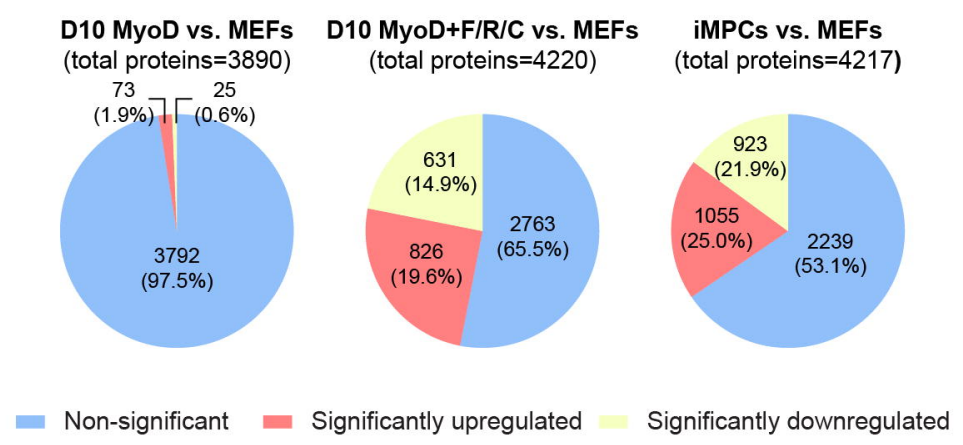

C

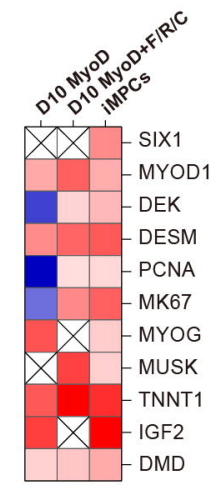

$\log 2 \mathrm{FC}$ (vs. MEFs)

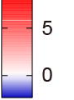

D
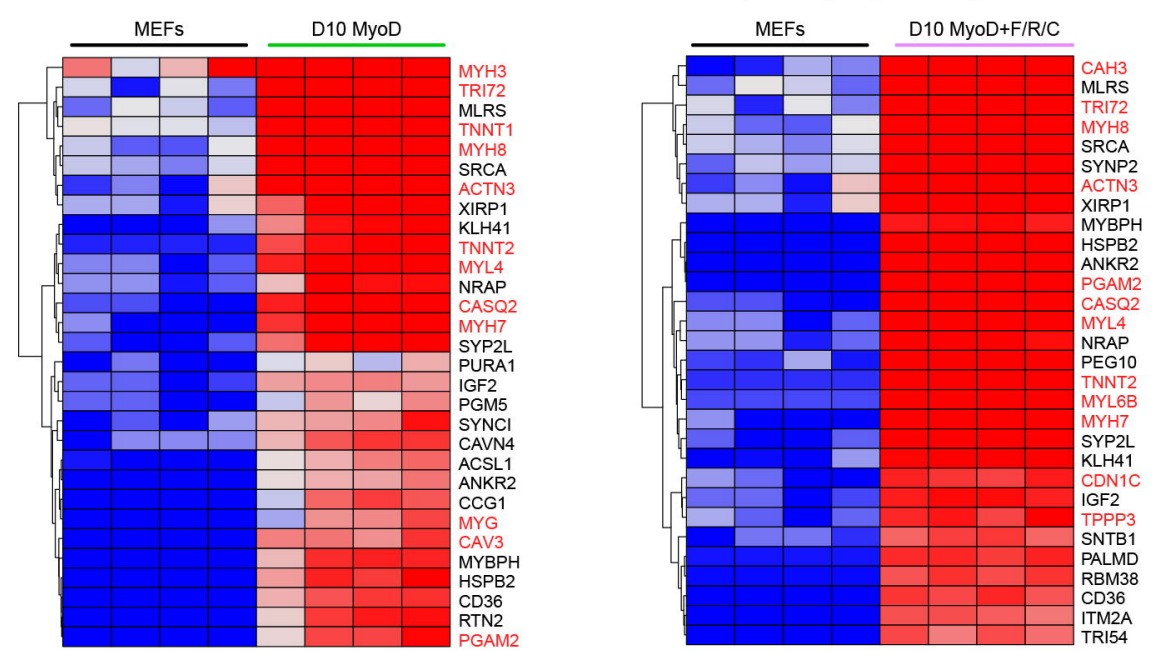

E

D10 MyoD+F/R/C vs. D10 MyoD

(total proteins $=4097$ )

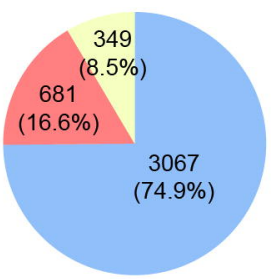

Non-significant

Significantly upregulated

Significantly downregulated
$\mathbf{F}$

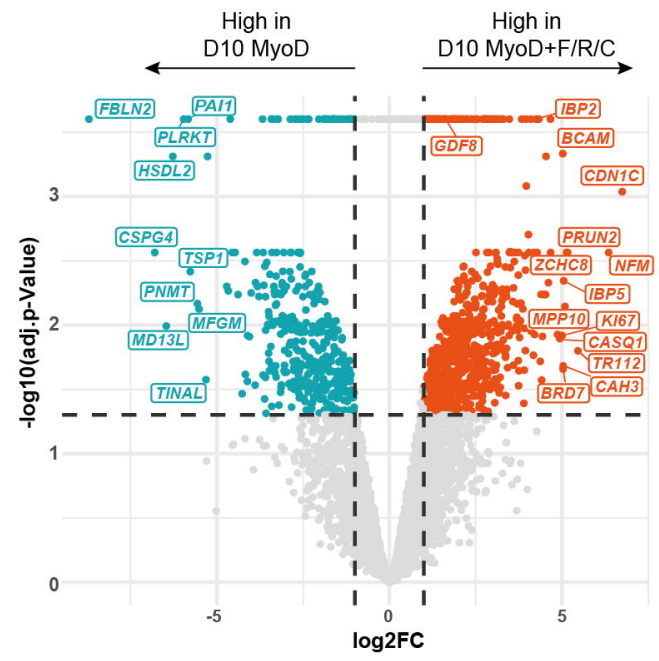

$\mathrm{H}$

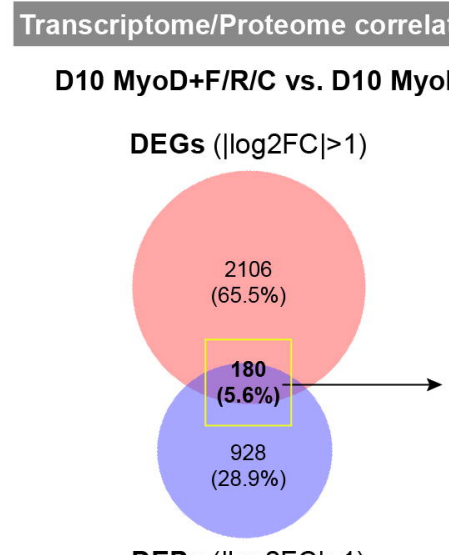

DEPs $(|\log 2 \mathrm{FC}|>1)$

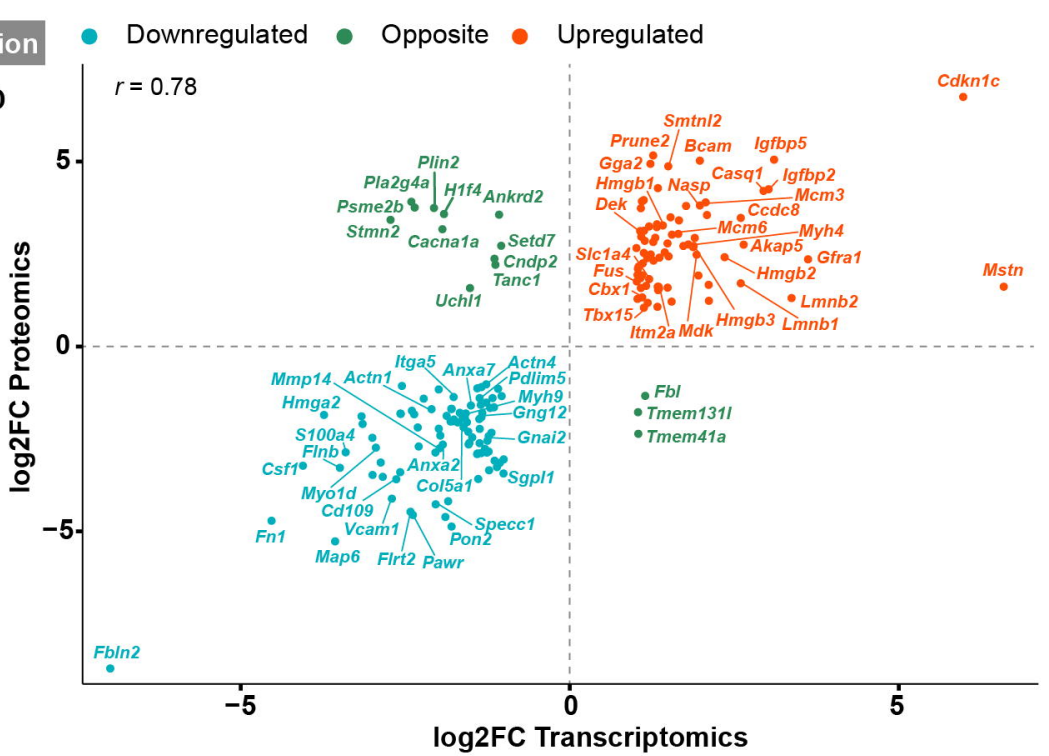

G

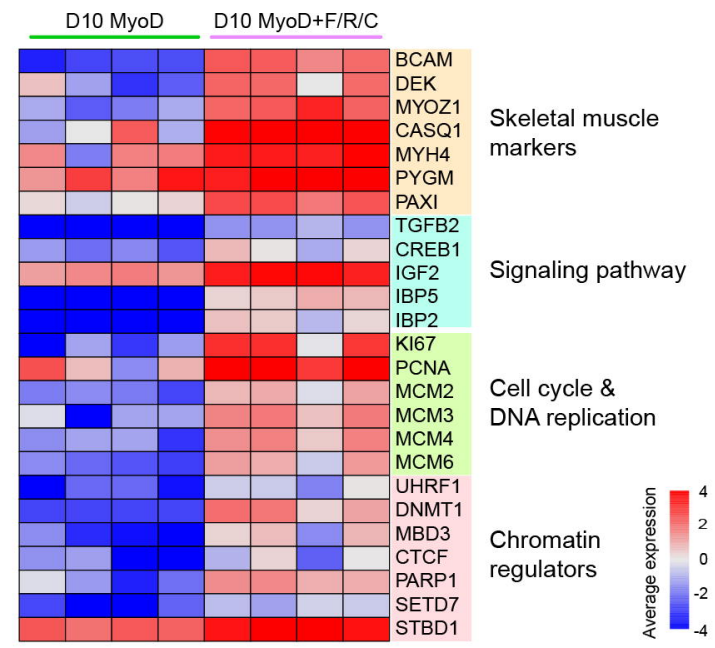

Enrichment analysis

(process network)

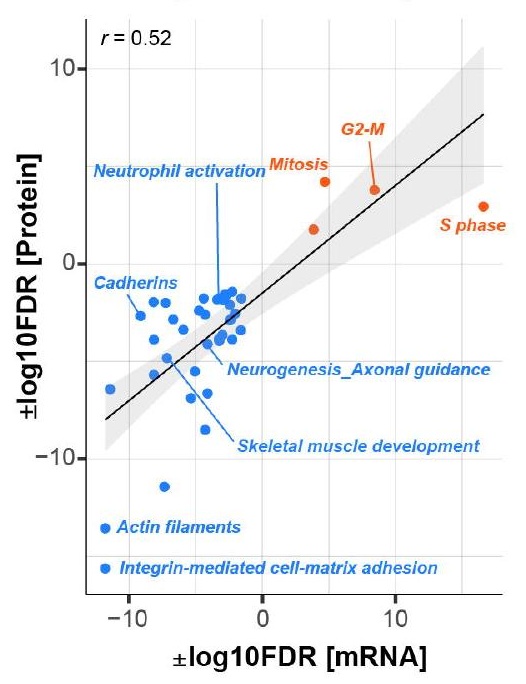




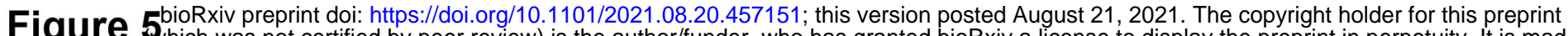
A the author/funder, who has granted bioRxiv a license to display the preprint in perpetuity. It is made available under aCC-BY-NC-ND 4.0 International liBense.

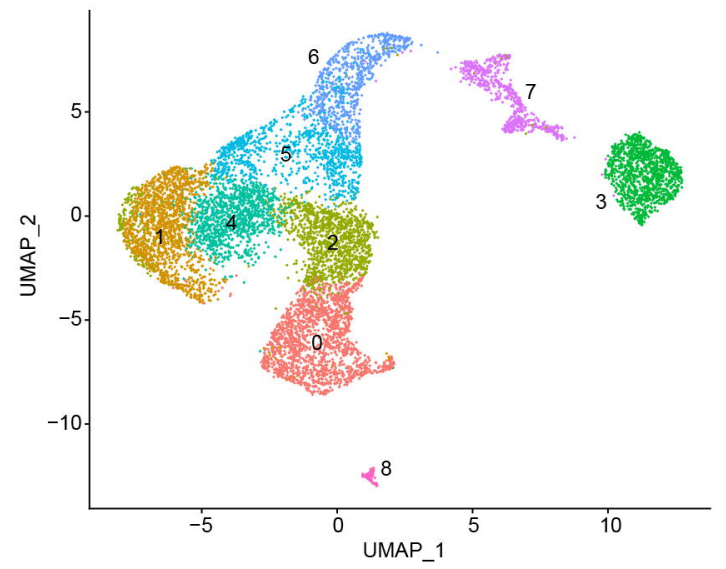

C

C
Pax7

E

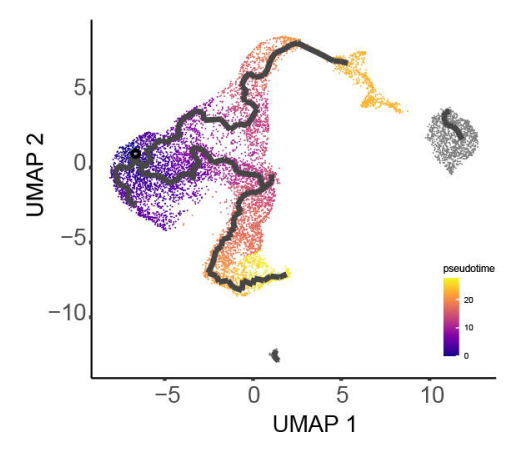
- Cycling Pax $7^{+}$progenitors $\left(\mathrm{Ccnb} 1^{+}, \mathrm{Mki}^{+} 7^{+}\right.$ - Pax7+ progenitors (Ccnb1-, Mki67-) - Fbln2+ fibroblasts

- Pax $7^{+}$progenitors (Ccnb1-, Mki67 $7^{+}$

Committed progenitors (Myod1 ${ }^{+}$, Myog $^{+}$)

- Myocytes (Myod1+, Myog ${ }^{+}, \mathrm{MyHC}^{+}$)

- Mature myofibers $\left(\mathrm{Tn}^{+}, \mathrm{MyHC}^{+}\right)$

- Cells expressing mesenchymal progenitor markers
Cells expressing connective tissue markers

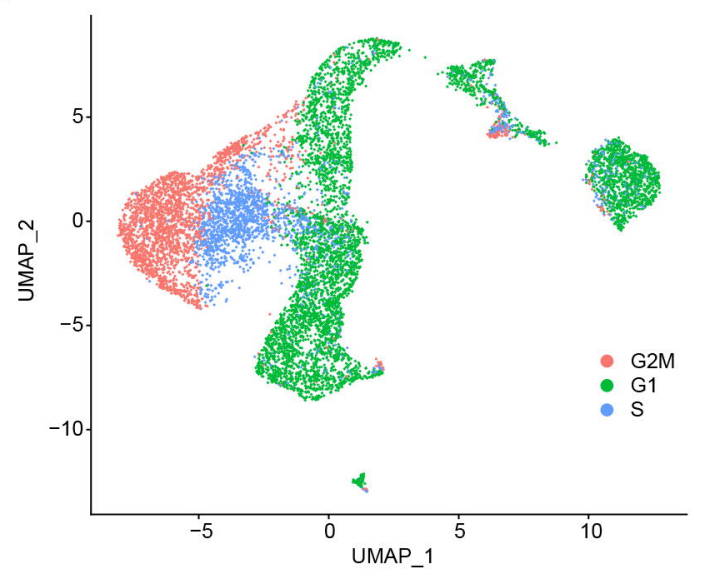

D
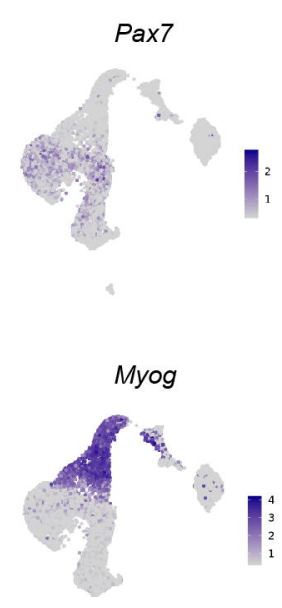

Dek

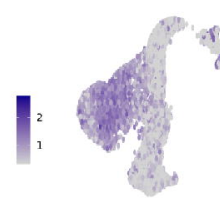

Sox8

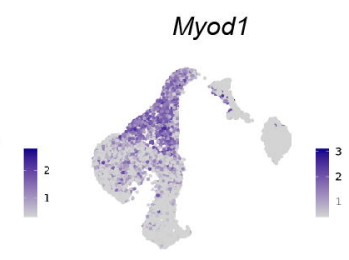

$\mathbf{F}$

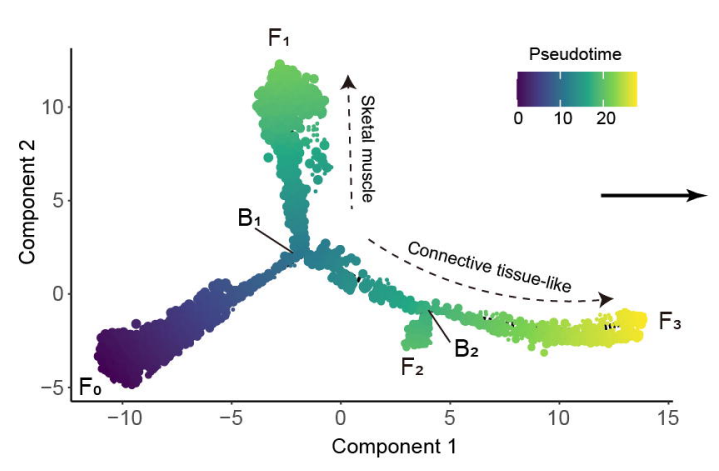

G

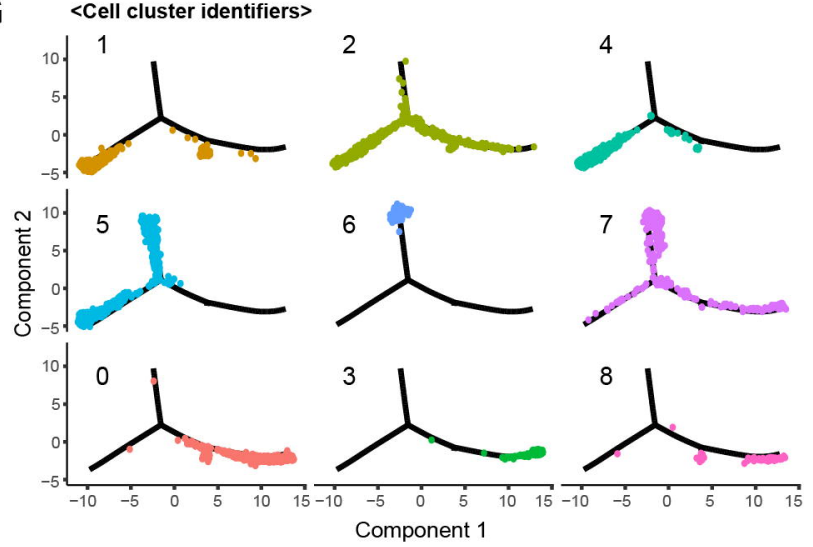

H
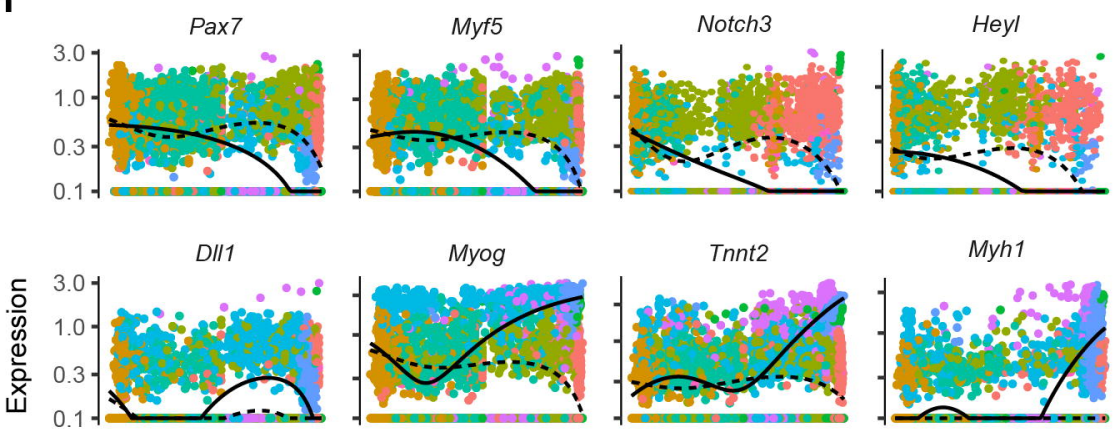

$-F$

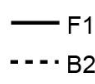

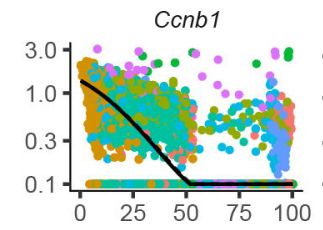

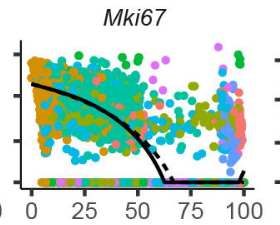

Fbln2
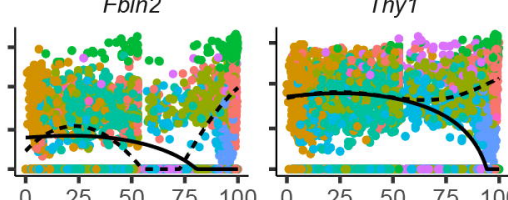

Pseudotime (stretched)

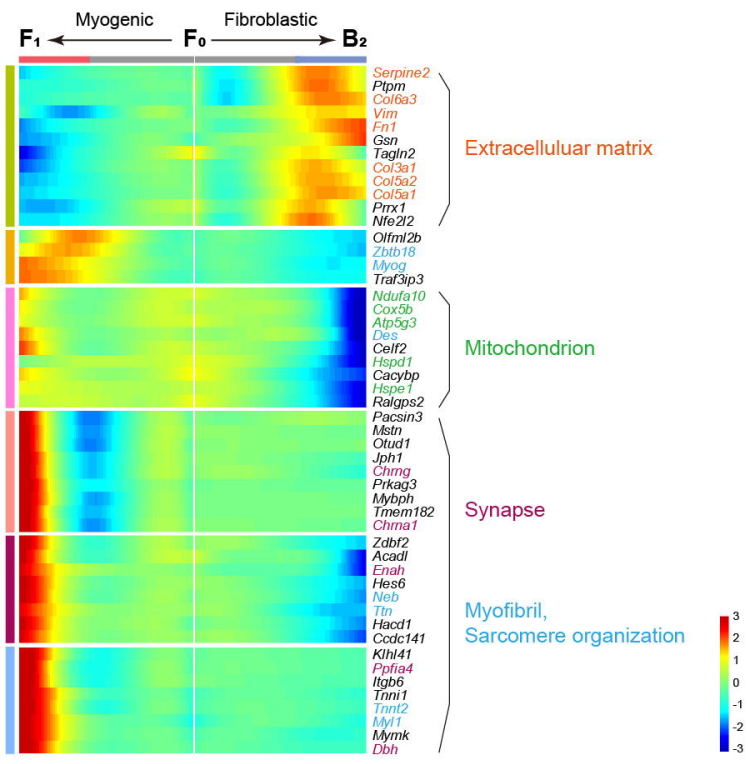


Figure GioRxiv preprint doi: https://doi.org/10.1101/2021.08.20.457151; this version posted August 21,2021 . The copyright holder for this preprint

A

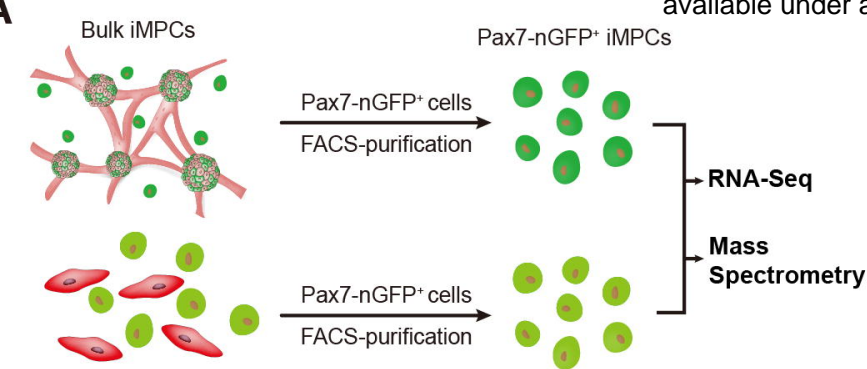
available under aCCBY-NC-ND 4.0 International license.

Primary myoblasts (e: Pax7 myocytes)

Pax7-nGFP+ myoblasts

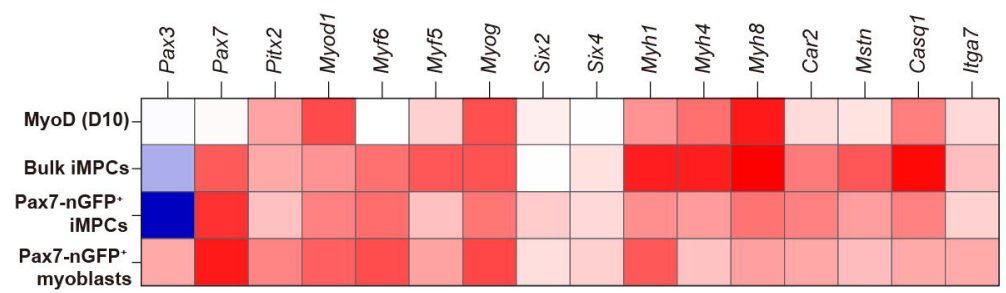

$\log 2 \mathrm{FC}$ (vs. MEFs)

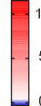

C

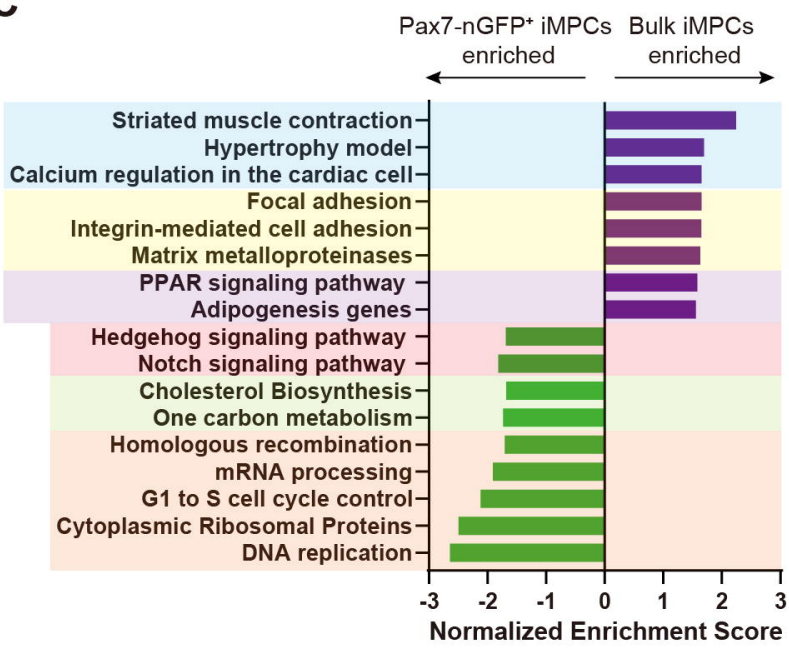

$\mathbf{F}$

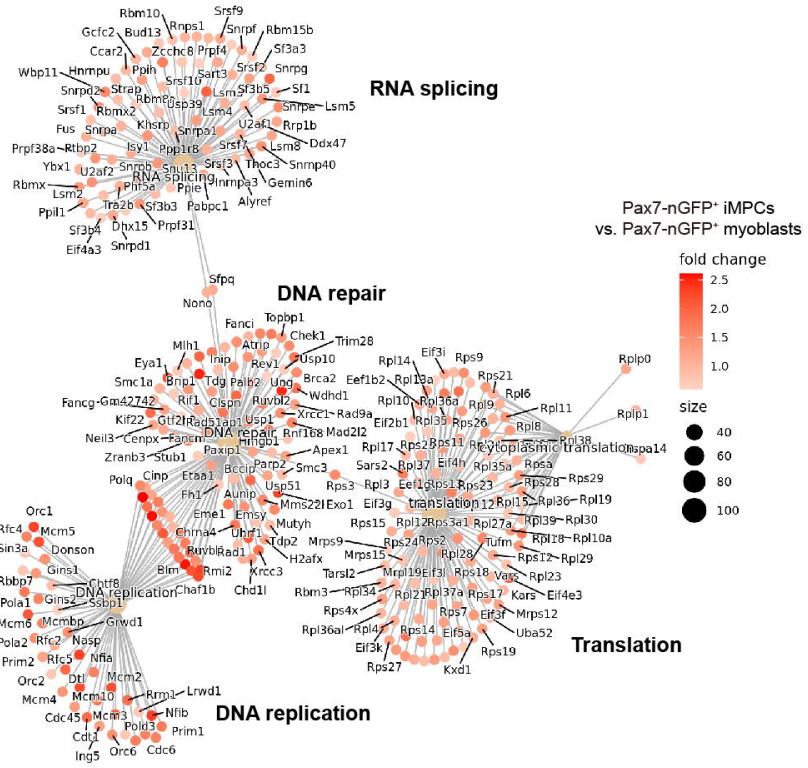

D

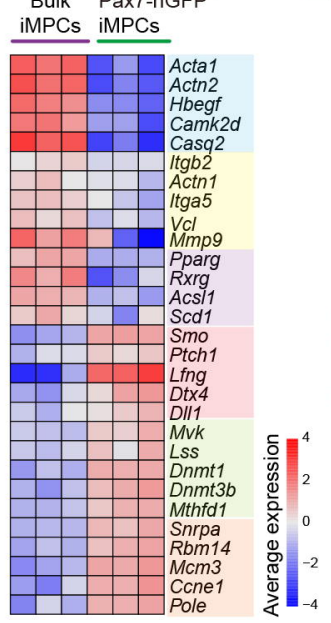

G

Satellite cell-specific markers

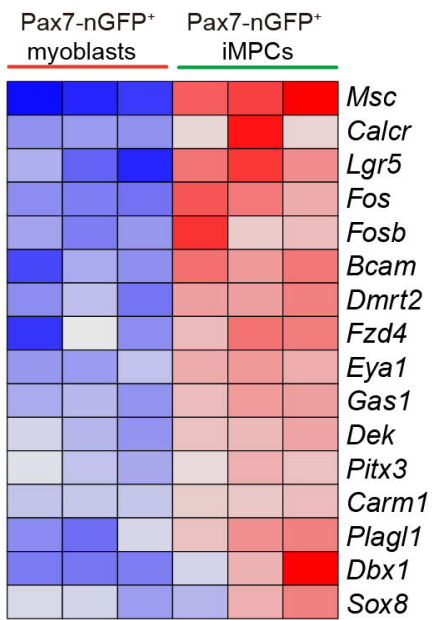

E

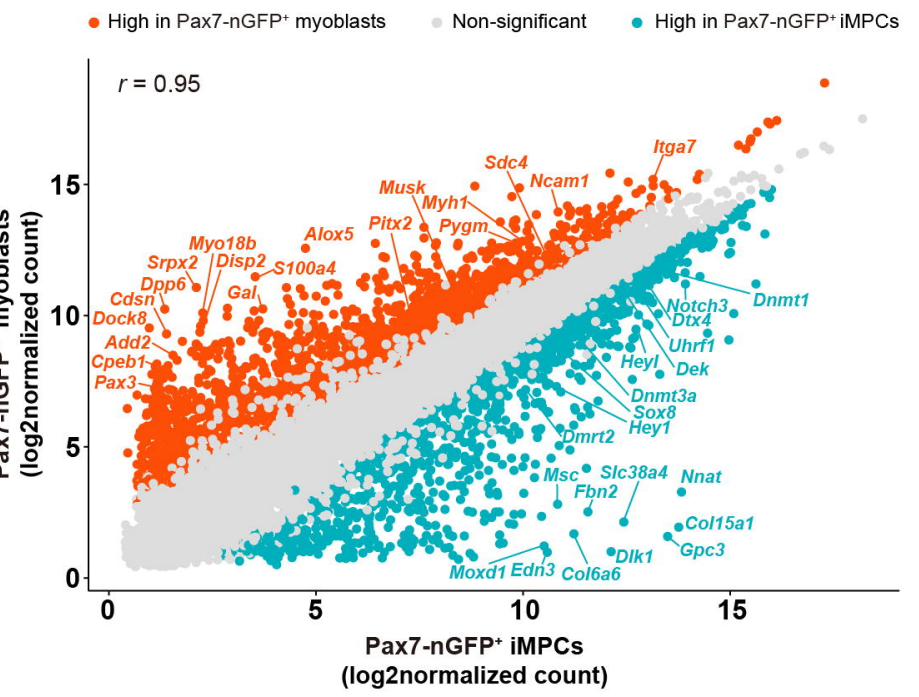

H

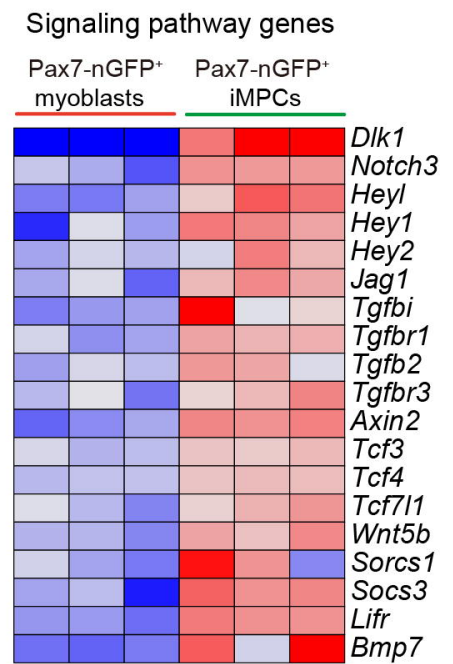

I

J

Pax7-nGFP ${ }^{+}$myoblasts Pax7-nGFP+ iMPCs $^{-}$

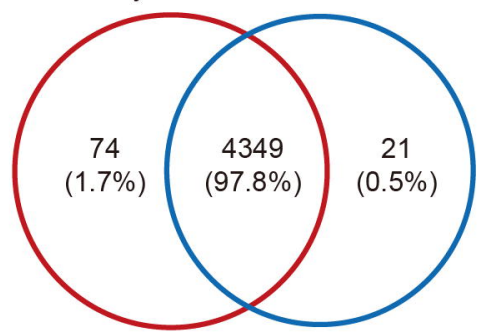

\# Proteins detected
Pax7-nGFP+ myoblasts vs. Pax7-nGFP' iMPCs

(total proteins $=\mathbf{4 4 4 4 )}$

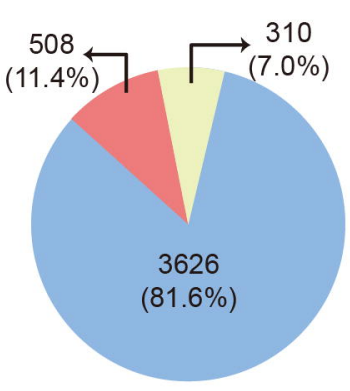

- non-significant

- High in Pax7-nGFP+ myoblasts High in Pax7-nGFP+ iMPCs
K

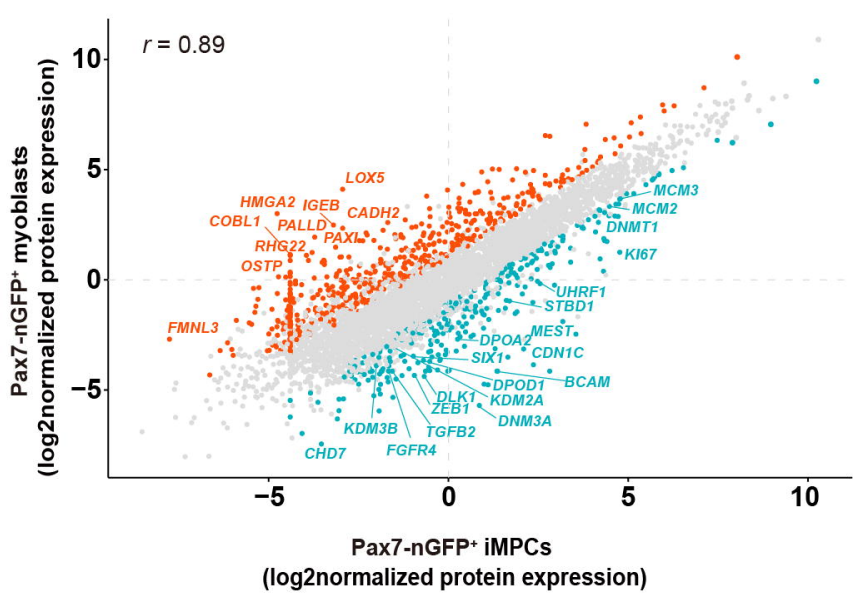


Figure 7bioRxiv preprint doi: https://doi.org/10.1101/2021.08.20.457151; this version posted August 21, 2021. The copyright holder for this preprint A available under aCC-BY-NC-ND 4.0 International license. $\begin{array}{cc}\text { Heyl } & \text { Notch1 } \\ \text { Notch3 }\end{array}$
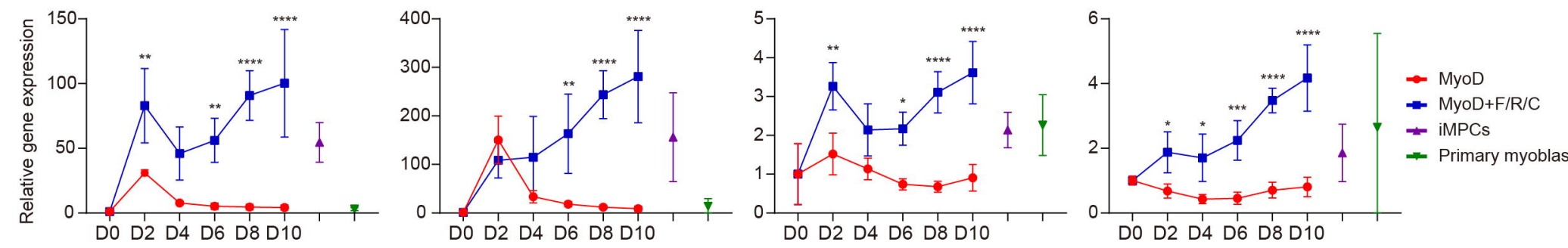

B

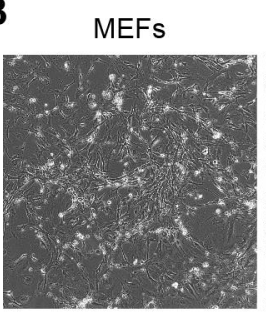

C

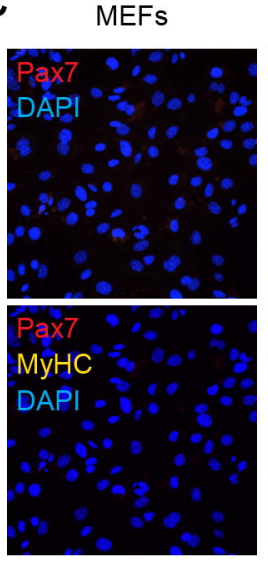

E

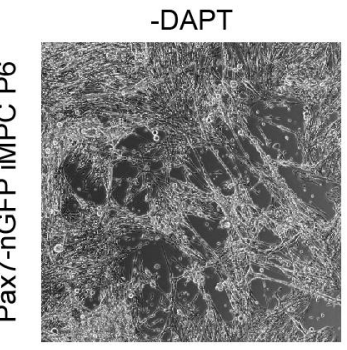

MyoD

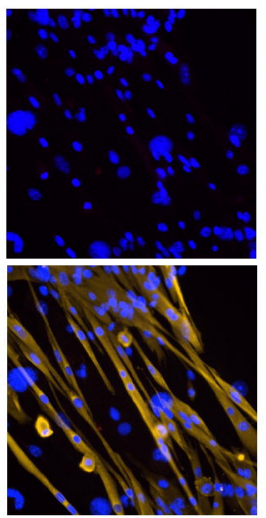

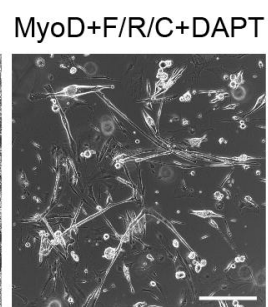

MyoD+F/R/C+DAPT
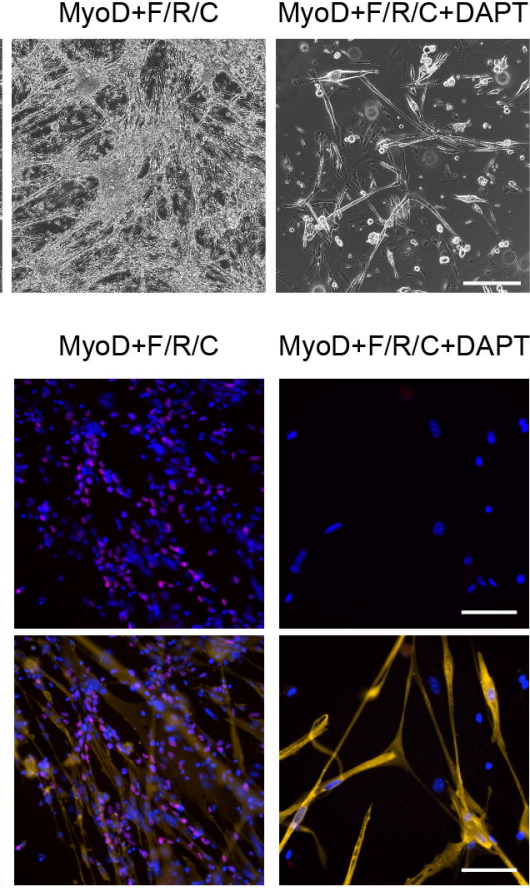
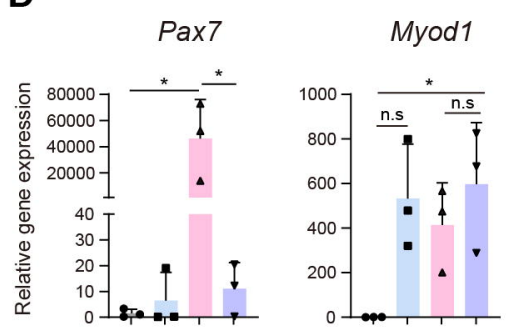

Notch1

Hey1

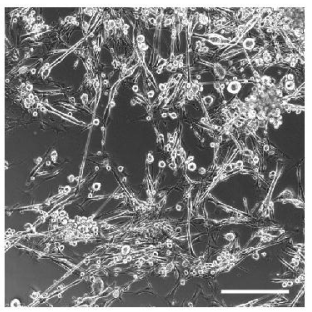

$\mathrm{H}$

吕
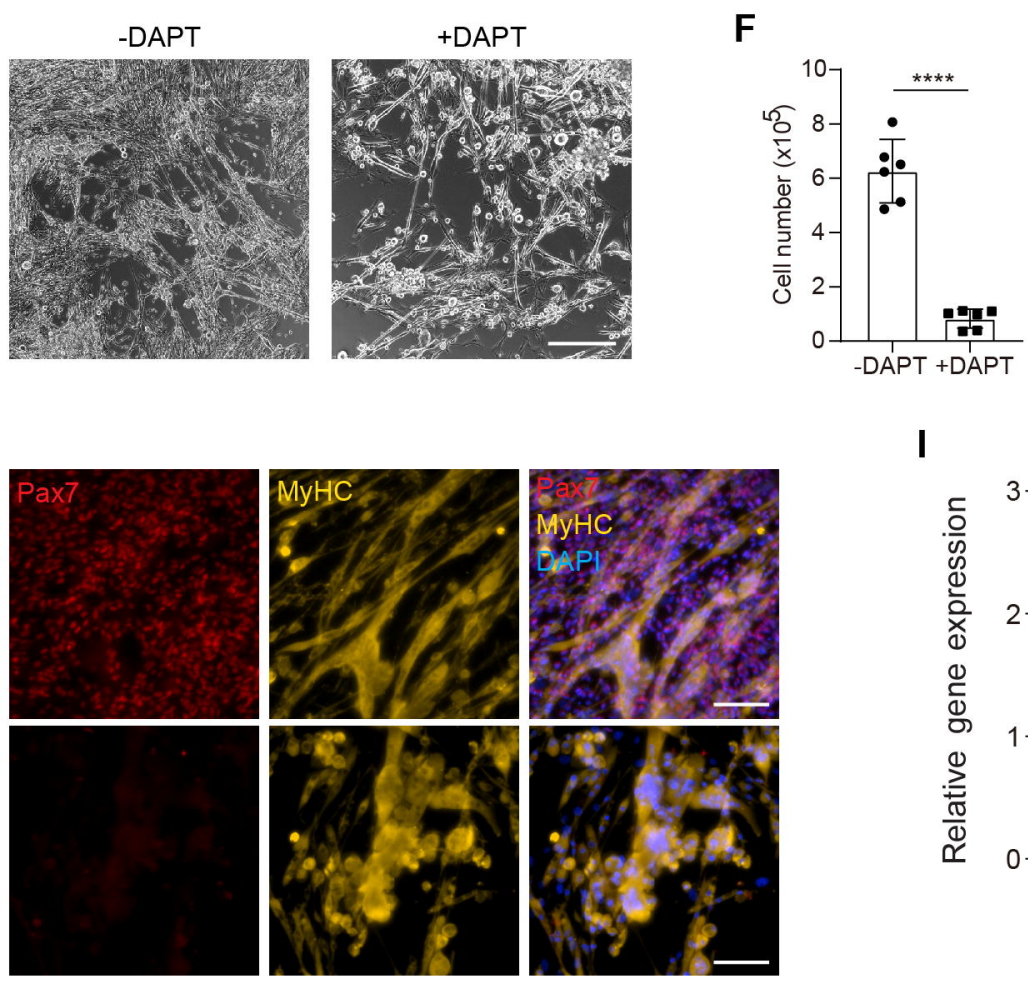
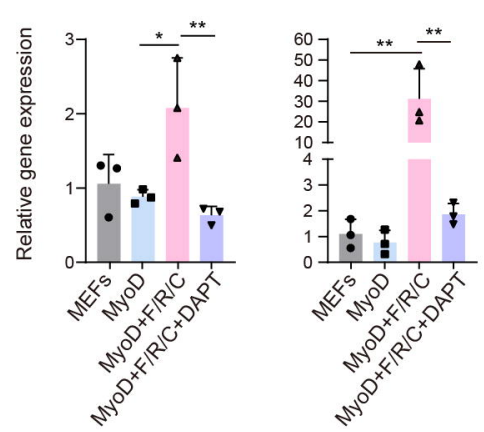

G
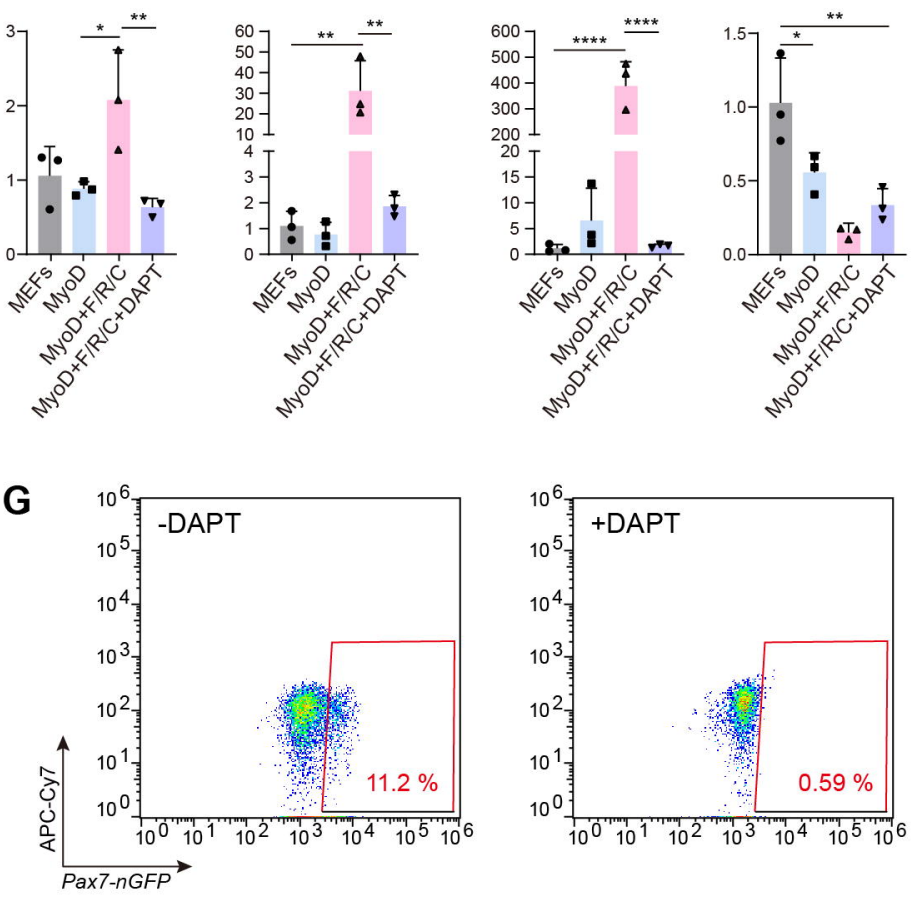

I

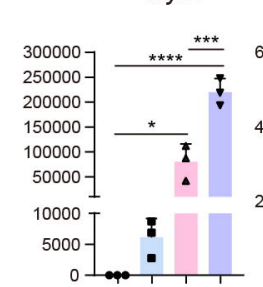

MyHC1

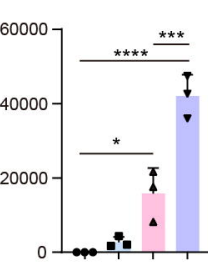

Thy1

\section{J}

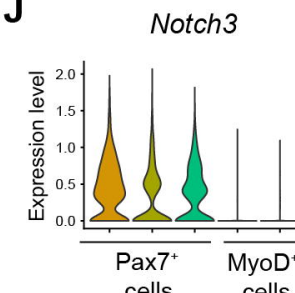

Hey1

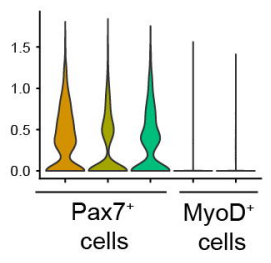

Heyl

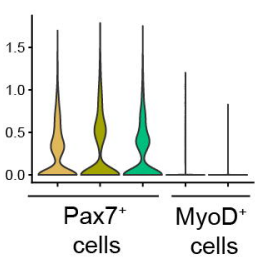

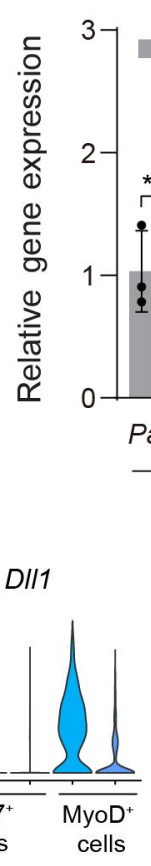

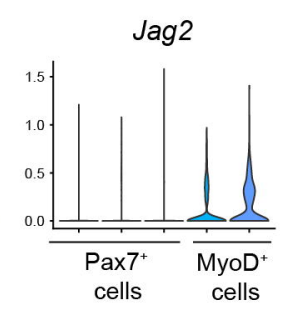

Hes6

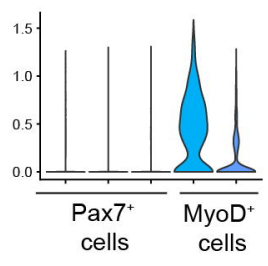

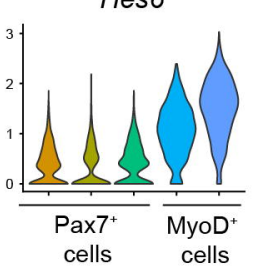

Cokn1c

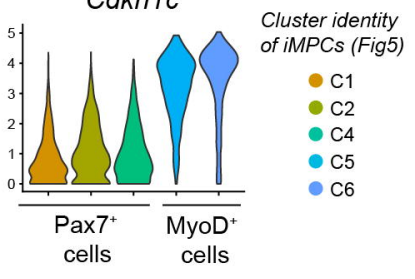

